\title{
Synergistic and Offset Effects of Fungal Species Combinations on Plant Performance
}

\section{OPEN ACCESS}

Edited by:

Angel Valverde,

Consejo Superior de Investigaciones

Cientificas (CSIC), Spain

Reviewed by:

Hassan Etesami,

University of Tehran, Iran

Casper Kamutando,

University of Zimbabwe, Zimbabwe

Errol Duncan Cason,

University of the Free State,

South Africa

*Correspondence:

Hirokazu Toju

toju.hirokazu.4c@kyoto-u.ac.jp

${ }^{\dagger}$ These authors have contributed

equally to this work

Specialty section:

This article was submitted to

Systems Microbiology,

a section of the journal

Frontiers in Microbiology

Received: 22 May 2021

Accepted: 20 August 2021

Published: 13 September 2021

Citation:

Hori Y, Fujita H, Hiruma K,

Narisawa K and Toju H (2021)

Synergistic and Offset Effects

of Fungal Species Combinations on

Plant Performance.

Front. Microbiol. 12:713180.

doi: 10.3389/fmicb.2021.713180

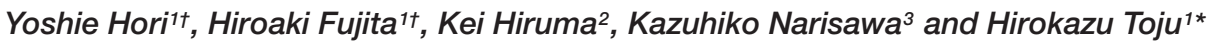

${ }^{1}$ Center for Ecological Research, Kyoto University, Kyoto, Japan, ${ }^{2}$ Graduate School of Arts and Sciences, Multi-Disciplinary Sciences Life Sciences, The University of Tokyo, Tokyo, Japan, ${ }^{3}$ College of Agriculture, Ibaraki University, Mito, Japan
\end{abstract}

In natural and agricultural ecosystems, survival and growth of plants depend substantially on residing microbes in the endosphere and rhizosphere. Although numerous studies have reported the presence of plant-growth promoting bacteria and fungi in below-ground biomes, it remains a major challenge to understand how sets of microbial species positively or negatively affect plants' performance. By conducting a series of single- and dual-inoculation experiments of 13 plant-associated fungi targeting a Brassicaceae plant species (Brassica rapa var. perviridis), we here systematically evaluated how microbial effects on plants depend on presence/absence of co-occurring microbes. The comparison of single- and dual-inoculation experiments showed that combinations of the fungal isolates with the highest plant-growth promoting effects in single inoculations did not have highly positive impacts on plant performance traits (e.g., shoot dry weight). In contrast, pairs of fungi with small/moderate contributions to plant growth in single-inoculation contexts showed the greatest effects on plants among the 78 fungal pairs examined. These results on the offset and synergistic effects of pairs of microbes suggest that inoculation experiments of single microbial species/isolates can result in the overestimation or underestimation of microbial functions in multispecies contexts. Because keeping single-microbe systems under outdoor conditions is impractical, designing sets of microbes that can maximize performance of crop plants is an important step for the use of microbial functions in sustainable agriculture.

Keywords: biodiversity, endophytes, microbial functions, species interactions, symbiosis, plant-associated microbiomes, plant-fungus interactions, microbe-microbe interactions

\section{INTRODUCTION}

Plants in natural and agricultural ecosystems are associated with diverse taxonomic groups of microbes, forming both positive and negative interactions with the microbiomes (Lundberg et al., 2012; Peay et al., 2016; Busby et al., 2017; Toju et al., 2018b). In particular, bacteria and fungi found within and around root systems have been reported as key determinants of plants' survival and growth (Hiruma et al., 2016, 2018; Castrillo et al., 2017; Trivedi et al., 2020). A number of rhizosphere bacteria, for example, are known to stimulate plants' growth by producing phytohormones (Lugtenberg and Kamilova, 2009; Bhattacharyya and Jha, 2012; Finkel et al., 2020). Mycorrhizal fungi are ancient symbionts of land plants (Remy et al., 1994; Taylor et al., 1995), providing soil phosphorus and/or nitrogen to their hosts (Richardson et al., 2009; 
Tedersoo et al., 2010; Jansa et al., 2019). Moreover, a growing number of studies have shown that diverse clades of endophytic and soil fungi support host plants by provisioning inorganic/organic forms of nutrients (Usuki and Narisawa, 2007; Newsham, 2011; Hiruma et al., 2016), activating plant immune systems (van Wees et al., 2008; Pieterse et al., 2014), and suppressing populations of pathogens/pests in the rhizosphere (Narisawa et al., 2004; Khastini et al., 2012; Gu et al., 2020). Thus, developing scientific bases for maximizing the benefits from those plant-associated microbiomes is an essential step for fostering sustainable crop production and restoring forest/grassland ecosystems (Bulgarelli et al., 2013; Carlström et al., 2019; Wagg et al., 2019; Saad et al., 2020).

One of the major challenges in utilizing plant-associated microbial functions is to design sets of microbial species/isolates (Vorholt et al., 2017; Paredes et al., 2018; Toju et al., 2018a; Wei et al., 2019). While a single microbial species or isolate can have specific functions in promoting plant growth, broader ranges of positive effects on plants are potentially obtained by introducing multiple microbial species/isolates (Wang et al., 2011; Ważny et al., 2018; He et al., 2020). For example, a fungal species degrading organic nitrogen (Newsham, 2011) and another fungus suppressing soil pathogens (Vinale et al., 2008) may collectively provide plants with a broader spectrum of physiological functions than each of them alone, potentially having additive or synergistic effects on the growth of their hosts. Meanwhile, sets of microbes trying to colonize the plant endosphere or rhizosphere may compete for resources/space (Kennedy et al., 2009; Werner and Kiers, 2015; Toju et al., 2016) or inhibit each other's growth (Helfrich et al., 2018), making their impacts on host plants more negative than that observed under single-inoculation conditions (i.e., offset effects) (Nelson et al., 2018). Given that multiple microbial species inevitably interact with a single plant in agroecosystems (Toju et al., 2018a), knowledge of those synergistic and offset effects in plant-associated microbiomes is crucial for optimizing microbial functions in crop production.

A starting point for designing sets of microbes is to use the information of single-inoculation assays, in each of which a single microbial species/isolate is introduced to a target plant species/variety (Ahmad et al., 2008; Harbort et al., 2020). Through this initial assay, respective species/isolates are scored in terms of their functions (e.g., plant-growth promotion effects) under single-inoculation conditions (Nara, 2006; Dai et al., 2008; Taurian et al., 2010; Tsolakidou et al., 2019). The next step is to consider how these single-inoculation scores can be used for designing sets of microbes that potentially promote plant growth in synergistic ways. As the number of combinations inflates with that of constituent species/isolates [e.g., $\{\mathrm{N} \times(\mathrm{N}-1)\} / 2$ combinations in two-species systems], prioritizing candidate species/isolate combinations based on single-inoculation results is an important step (Paredes et al., 2018; Toju et al., 2018a, 2020). The simplest way of exploring best sets of microbes is to combine microbes with highest single-inoculation scores. This strategy of combining microbes in highest ranks is promising if synergistic (or additive) effects are common in plant-associated microbiomes. In contrast, if offset effects of multiple microbes on plant performance are ubiquitous, alternative strategies for exploring species/isolate combinations are required to maximize benefits from plant-associated microbiomes. Thus, knowledge of the prevalence and intensity of such synergistic and offset effects is essential in synthetic microbiome studies. Nonetheless, although there have been studies reporting synergistic/offset effects of multiple plant-associated microbes (Han and Lee, 2006; Wang et al., 2011; Ważny et al., 2018; He et al., 2020), experimental studies systematically evaluating the commonness of those effects are scarce.

In this study, we tested the hypothesis that synergistic effects on plant growth are common in below-ground fungal biomes in a series of single- and dual-inoculation experiments. By using 13 plant-associated fungal species belonging to various taxonomic groups, we first evaluated their basic effects on plant growth in a single-inoculation experiments with a Brassicaceae species (Brassica rapa var. perviridis). We also performed dualinoculation experiments for all the 78 possible combinations of the fungal species and then evaluated the performance of the combinations in light of single-inoculation results. The data then provided a platform for testing whether plant-growth promoting effects exceeding those of all the single-inoculation conditions are attainable under dual-inoculation conditions. Overall, this study provides a basis for understanding to what extent plant-growth promotion effects of microbiomes can be expected from the information of single-species inoculations, illuminating the potential importance of "non-additivity" in multi-microbe contexts.

\section{MATERIALS AND METHODS}

\section{Fungal Isolates for Inoculation Experiments}

In the inoculation experiments detailed below, we used diverse fungal species isolated from plant roots (Table 1). Among the 13 fungal isolates used (Table 1 and Supplementary Data 1), some are reported as endophytic fungi promoting plant growth [e.g., Colletotrichum tofieldiae, Cladophialophora chaetospira, and Veronaeopsis simplex] in previous studies (Usuki and Narisawa, 2007; Hiruma et al., 2016; Guo et al., 2018). In addition, a species of Trichoderma with growth-promotion effects on tomato (Solanum lycopersicum) and Brassica plants (Toju et al., 2020) was used in the experiment. In addition, various taxonomic groups of fungal isolates were retrieved from the ca. 3,500 fungal isolates maintained in the culture collection of Centre for Ecological Research, Kyoto University (Supplementary Data 1). The 13 isolates examined in this study were selected so that not only well-characterized plant-growth-promoting fungi but also fungi with potentially negative or nearly neutral effects on plants were targeted in the experiments.

In addition to observation under a microscope, the fungal isolates were identified based on the DNA sequencing of the internal transcribed spacer (ITS) region (DDBJ/ENA/NCBI accession number; LC632034-LC632046): all the ITS sequences matched NCBI database sequences of known fungal species with E-values less than $9.0 \times 10^{23}$ (Table 1 and Supplementary Data 1). Putative functional groups of these fungi were inferred 
TABLE 1 | Summary of fungal isolates used in the inoculation experiments.

\begin{tabular}{|c|c|c|c|c|c|c|c|c|c|c|c|}
\hline Isolate & Abbreviation & Phylum & Class & Order & Family & Genus & Guild & Blast top-hit & E value & Per. Ident & Accession \\
\hline $\begin{array}{l}\text { Phoma sp. } \\
\text { KUCER00000052 }\end{array}$ & pho_0052 & Ascomycota & Dothideomycetes & Pleosporales & Didymellaceae & Phoma & PES & Phoma leveillei & $9.00 E-123$ & $99.6 \%$ & $\underline{K Y 827373.1}$ \\
\hline $\begin{array}{l}\text { Alternaria sp. } \\
\text { KUCER00001239 }\end{array}$ & alt_1239 & Ascomycota & Dothideomycetes & Pleosporales & Periconiaceae & Alternaria & PES & Alternaria broccoli-italicae & $2.00 \mathrm{E}-123$ & $100.0 \%$ & $\underline{\mathrm{MH} 374617.1}$ \\
\hline $\begin{array}{l}\text { Curvularia sp. } \\
\text { KUCER00000077 }\end{array}$ & cur_0077 & Ascomycota & Dothideomycetes & Pleosporales & Periconiaceae & Curvularia & $P$ & Curvularia coatesiae & $4.00 \mathrm{E}-126$ & $100.0 \%$ & MK804384.1 \\
\hline $\begin{array}{l}\text { Setosphaeria sp. } \\
\text { KUCER00000031 }\end{array}$ & set_0031 & Ascomycota & Dothideomycetes & Pleosporales & Periconiaceae & Setosphaeria & PE & Setosphaeria pedicellata & $1.00 \mathrm{E}-126$ & $100.0 \%$ & LT837452.1 \\
\hline $\begin{array}{l}\text { Stemphylium sp. } \\
\text { KUCER00000804 }\end{array}$ & ste_0804 & Ascomycota & Dothideomycetes & Pleosporales & Periconiaceae & Stemphylium & PS & Stemphylium lycopersici & $2.00 \mathrm{E}-125$ & $100.0 \%$ & MN386223.1 \\
\hline $\begin{array}{l}\text { Veronaeopsis } \\
\text { simplex Y34 }\end{array}$ & ver_0232 & Ascomycota & Dothideomycetes & Venturiales & Sympoventuriaceae & Veronaeopsis & E & Veronaeopsis simplex & $5.00 \mathrm{E}-125$ & $100.0 \%$ & MH865233.1 \\
\hline $\begin{array}{l}\text { Cladophialophora } \\
\text { chaetospira M4006 }\end{array}$ & cla_0230 & Ascomycota & Eurotiomycetes & Chaetothyriales & Herpotrichiellaceae & Cladophialophora & E & Cladophialophora chaetospira & $3.00 \mathrm{E}-123$ & $99.6 \%$ & $\underline{\underline{L C} 077702.1}$ \\
\hline $\begin{array}{l}\text { Aspergillus sp. } \\
\text { KUCER00000917 }\end{array}$ & asp_0917 & Ascomycota & Eurotiomycetes & Eurotiales & Aspergillaceae & Aspergillus & $\mathrm{s}$ & Aspergillus terreus & $7.00 \mathrm{E}-124$ & $99.6 \%$ & $\underline{\mathrm{MH} 124236.1}$ \\
\hline $\begin{array}{l}\text { Colletotrichum } \\
\text { tofieldiae MAFF } \\
712334\end{array}$ & col_0223 & Ascomycota & Sordariomycetes & Glomerellales & Glomerellaceae & Colletotrichum & PE & Colletotrichum tofieldiae & $2.00 E-125$ & $100.0 \%$ & $\underline{K X 069824.1}$ \\
\hline $\begin{array}{l}\text { Trichoderma sp. } \\
\text { KUCER00000218 }\end{array}$ & tri_0218 & Ascomycota & Sordariomycetes & Hypocreales & Hypocreaceae & Trichoderma & PFES & Trichoderma asperellum & $5.00 \mathrm{E}-125$ & $100.0 \%$ & MT530021.1 \\
\hline $\begin{array}{l}\text { Fusarium sp. } \\
\text { KUCER00000983 }\end{array}$ & fus_0983 & Ascomycota & Sordariomycetes & Hypocreales & Nectriaceae & Fusarium & PES & Fusarium oxysporum & $5.00 \mathrm{E}-125$ & $100.0 \%$ & MT610995.1 \\
\hline $\begin{array}{l}\text { Tolypocladium sp. } \\
\text { KUCER00000289 }\end{array}$ & tol_0289 & Ascomycota & Sordariomycetes & Hypocreales & Ophiocordycipitaceae & Tolypocladium & $\mathrm{FE}$ & Tolypocladium album & $9.00 \mathrm{E}-123$ & $99.6 \%$ & $\underline{\text { LC386577.1 }}$ \\
\hline $\begin{array}{l}\text { Mucor sp. } \\
\text { KUCERO0000113 }\end{array}$ & muc_0113 & Mucoromycota & - & Mucorales & Mucoraceae & Mucor & S & Mucor abundans & $1.00 \mathrm{E}-125$ & $100.0 \%$ & MK164195.1 \\
\hline
\end{tabular}

For each fungal isolate, taxonomy, functional guild information inferred by the FUNGuild database (P, plant pathogen; F, fungal pathogen; E, endophyte; S, saprophyte), and NCBI BLAST top-hit results of the ITS sequences are indicated for each isolate. See Supplementary Data 1 for detailed information of the isolates. 
based on the taxonomic information using the FUNGuild program (Nguyen et al., 2016) as shown in Table 1. Note that such profiling information based on ecological guild databases should be interpreted with caution: even in a fungal genus embracing a number of plant pathogenic species, some species can have positive impacts on plants (Radhakrishnan et al., 2015; Hiruma et al., 2016).

\section{Fungal Inocula}

Prior to the inoculation experiments, fungal inocula were prepared by modifying the protocols of some previous studies (Harsonowati et al., 2020; Toju et al., 2020) as detailed below. Each of the 1.3-L high-density polyethylene bags with airconditioning filters (Shinkon Co. Ltd., Minokamo) was filled with the mixture of $60-\mathrm{cm}^{3}$ wheat bran (Tamagoya Shoten), 60$\mathrm{cm}^{3}$ rice bran, $180-\mathrm{cm}^{3}$ leaf mold (Akagi Gardening Co., Ltd.), and $70-\mathrm{mL}$ distilled water. The filled culture bags were sealed with a heat sealer (ANT-300, AS ONE Corporation, Osaka) and autoclaved three times at $121^{\circ} \mathrm{C}(103.7 \mathrm{kPa})$ for $30 \mathrm{~min}$ with $24 \mathrm{~h}$ intervals. For each fungal isolate, approximately ten pieces of mycelial disks $(8.0 \mathrm{~mm}$ in diameter) were then transferred from 1/2 CMMY medium (cornmeal agar, $8.5 \mathrm{~g} / \mathrm{L}$; malt extract, $10.0 \mathrm{~g} / \mathrm{L}$; yeast extract, $1.0 \mathrm{~g} / \mathrm{L}$ ) (Becton, Dickinson and Co.) to the autoclaved substrate and the fungal culture bag was incubated at room temperature (approximately $25^{\circ} \mathrm{C}$ ) for $10-21$ days until it was filled with mycelia. In addition to the 13 fungal inocula, a mock inoculum without fungi was prepared as a control.

Each of the fungal/control inocula was mixed with autoclaved potting soil [one round of $121^{\circ} \mathrm{C}(103.7 \mathrm{kPa})$ for $20 \mathrm{~min}$ ] consisting mainly of organic materials such as fermented bark, peat moss, and coconut peat ["Gin-no-tsuchi"; Total $\mathrm{N}, 4,100 \mathrm{mg} / \mathrm{kg} ; \mathrm{P}, 2,706 \mathrm{mg} / \mathrm{kg} ; \mathrm{K}, 2,823 \mathrm{mg} / \mathrm{kg} ; \mathrm{pH}, 6.70$; electrical conductivity, $0.73 \mathrm{mS} / \mathrm{cm}$; Kanea Inc., Takamatsu] by the proportion of 1:9. The mixed soil was then transferred into plastic cell trays: the size of each cell in the trays was $49 \mathrm{~mm} \times 49 \mathrm{~mm} \times 56.5 \mathrm{~mm}$.

\section{Inoculation Experiments}

The "Komatsuna Wase" variety of B. rapa var. perviridis (Atariya Noen Co. Ltd., Katori) was used as the target plant in the inoculation experiments because its fast-growing property was convenient for the assay. Before inoculation, the seeds of Brassica were surface sterilized by being shaken in $70 \%$ ethanol solution for $1 \mathrm{~min}$ and then in $1 \%$ sodium hypochlorite solution for $1.5 \mathrm{~min}$. The seeds were then rinsed three times in distilled water. They were subsequently placed on $1 \%$ agar petri dishes and incubated at $23^{\circ} \mathrm{C}$ in the dark for $24-26 \mathrm{~h}$ until rooting. The rooted seeds were transferred to the inoculum-mixed soil on the following day: two seeds were introduced into each of the 20-35 replicate cells for each single inoculation experiment (Supplementary Data 2). The cell trays were maintained in the laboratory with the $16 \mathrm{hL} / 8 \mathrm{hD}$ light condition at $25^{\circ} \mathrm{C}$. The plants were watered 3-4 times a week. The locations of the cell trays were rotated to equalize plants' growing conditions.

In addition to the above single-inoculation experiments, dualinoculation experiments were performed for all the 78 possible combinations of the 13 fungal isolates. For each pair of fungal isolates, their inocula were mixed by the proportion of $1: 1$, collectively constituting $1 / 10$ volume of the total soil volume within the cell pots. Two Brassica seeds were then introduced into each of the replicate cell pots and they were kept under laboratory conditions as detailed above. Due to the large number of treatments and replicates as well as the limited spatial capacity of the laboratory, the inoculation experiments were split into several experimental rounds (up to $13 \mathrm{single/dual/control}$ treatments per round; see Supplementary Data 2 for the information of experimental rounds). To take into account potential difference of micro-environmental conditions among the experimental rounds, a control (mock inoculum) treatment was included in every round in order to standardize plant growth responses throughout the study (see below for the calculation of a standardized growth index).

After 7 days, the ratio of geminating seeds to introduced seeds (i.e., germination rate) was recorded for each single/dual/control treatment. The seedlings were randomly thinned to one seedling per cell and they were kept under the same environmental conditions for another 2 weeks.

\section{Plants' Growth Responses}

The 21-day old Brassica plant samples were harvested to evaluate their responses to fungal inoculations. For all the replicate samples, shoot dry weight (above-ground biomass) and the number of mature leaves (>20 mm in length) were recorded. For the measurement of shoot dry weight, plant samples were oven-dried at $60^{\circ} \mathrm{C}$ for at least $72 \mathrm{~h}$. Leaves longer than $20 \mathrm{~mm}$ were also subjected to SPAD measurements to infer chlorophyll concentrations using a SPAD-502Plus meter (Konica Minolta, Inc., Tokyo) (Netto et al., 2005; Zhu et al., 2012). For each of the randomly-selected 15 plant samples per treatment, the SPAD readings at three points were averaged. While shoot dry weight and the number of mature leaves are metrics of plant total biomass, SPAD readings (chlorophyll concentrations) are often regarded as (weak) indicators of foliar nitrogen concentrations (Chang and Robison, 2003; Esfahani et al., 2008).

To standardize the variables representing plants' responses to fungal inoculations, we calculated a standardized growth index as follows:

$$
S G_{T}(i)=\frac{X_{T}(i)-\overline{X_{C}}}{S D_{C}}
$$

where $X_{T}(i)$ is a measurement of a target trait of a plant sample $i$ in a target single-/dual-inoculation treatment, while $\overline{X_{C}}$ and $S D_{C}$ are the mean and standard deviation of plant traits (variables) observed in the control samples of the focal experimental round, respectively. In terms of basic statistics assuming the Gaussian distribution, the standardized growth index $\left[S G_{T}(i)\right]$ values less than -1.96 and those larger than 1.96 roughly represented plant performance outside the $95 \%$ confidence intervals of the control samples in the same experimental rounds, providing an intuitive criterion for comparing results within/across inoculation experiments (see Supplementary Figure 1 for relationship between the standardized growth index values and false discovery rates). This standardized growth index was calculated for each of the three plant variables representing plant 
A Single-inoculation effects on plant performance
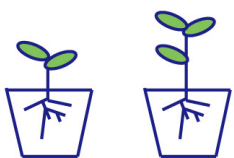

(1)

Control

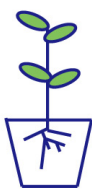

0
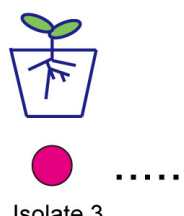
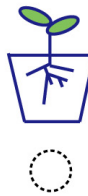

Control
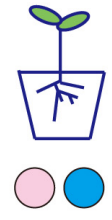

Isolate 1

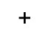

Isolate 2
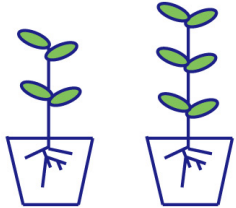

Isolate 2

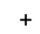

Isolate 3

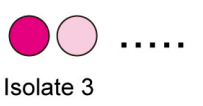

$+$

Isolate 1
B

Synergistic effects

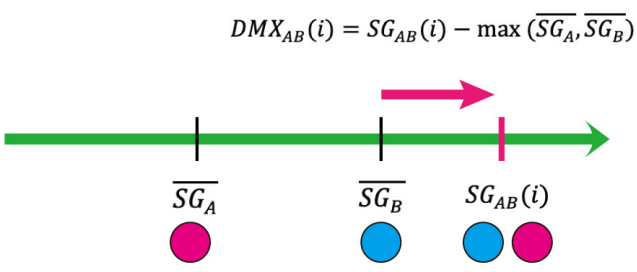

Deviation (single- vs. dual-inoculations)

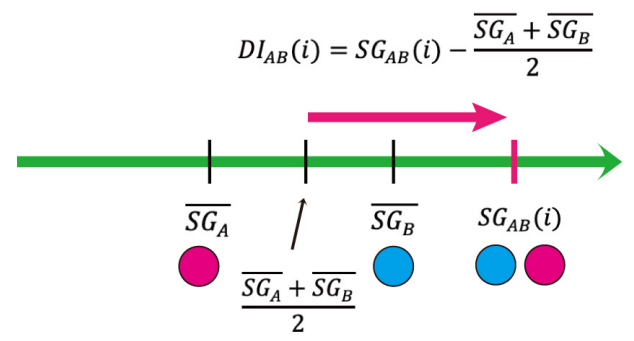

Offset effects

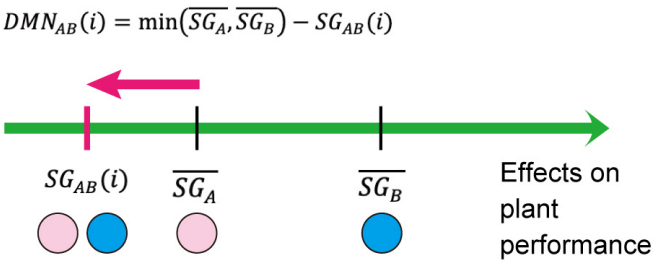

FIGURE 1 | Evaluation of effects on plant performance. (A) Schema of single- and dual-inoculation assays. (B) Indexes for comparing single vs. dual inoculation effects. Along the axis of standardized growth index defined by the Equation (1), index values representing synergistic/offset effects on plants are calculated for each replicate plant sample for each pair of microbial (fungal) isolates $\left[D M X_{A B}(i)\right.$ and $\left.D M N_{A B}(i)\right]$. Likewise, index values representing deviation of dual-inoculation effects from single-inoculation effects are obtained $\left[D I_{A B}(i)\right]$. (C) Example of inoculation experiments. Brassica plants inoculated with two fungal isolates (tri_0218 × ste_0804; right) and those without fungal inoculations (control; left).

performance: the number of mature leaves, shoot dry weight, and chlorophyll concentrations.

\section{Synergistic and Offset Effects}

Based on the standardized growth index, we evaluated potential synergistic effects in dual inoculations of two fungal isolates in comparison to single-inoculation results (Figure 1). For a replicate plant sample inoculated with a pair of fungal isolates $\mathrm{A}$ and $\mathrm{B}$, the index representing deviation from the maximum effects in single inoculations is calculated as follows:

$$
D M X_{A B}(i)=S G_{A B}(i)-\max \left(\overline{S G_{A}}, \overline{S G_{B}}\right),
$$

where $S G_{A B}(i)$ is the standardized growth index of a replicate plant in the dual inoculation treatment, while $\overline{S G_{A}}$ and $\overline{S G_{B}}$ are means of standardized growth index values for the single inoculation of fungal isolates A and B, respectively. By definition, when there are synergistic effects of the presence of two fungal isolates [i.e., $\left.\overline{S G_{A B}}>\max \left(\overline{S G_{A}}, \overline{S G_{B}}\right)\right]$, the mean of the deviation index over replicate plant samples $\left(\overline{D M X_{A B}}\right)$ is larger than zero. Likewise, to evaluate offset effects in dual inoculations of two fungal isolates, an index representing deviation from the minimum effects in single inoculations was defined as follows:

$$
D N_{A B}(i)=\min \left(\overline{S G_{A}}, \overline{S G_{B}}\right)-S G_{A B}(i)
$$

When there are offset effects [i.e., $\overline{S G_{A B}}<\min \left(\overline{S G_{A}}, \overline{S G_{B}}\right)$ ] for a focal pair of fungi, mean of the offset effect index over replicate samples $\left(\overline{D M N_{A B}}\right)$ is larger than zero.

We further developed a simple index for evaluating deviations of observed dual-inoculation results from those expected as intermediate results of single inoculations. For a pair of fungal isolates $\mathrm{A}$ and $\mathrm{B}$, the index for deviation from intermediate effects is calculated as follows:

$$
D I_{A B}(i)=S G_{A B}(i)-\frac{\overline{S G_{A}}+\overline{S G_{B}}}{2} .
$$


If the plant-growth promoting effects under the presence of two fungal isolates is close to what is expected as the intermediate results of the single inoculation assays of the two isolates, the index for deviation from intermediate effects $\left[D I_{A B}(i)\right]$ or its mean over replicate samples $\left(\overline{D I_{A B}}\right)$ is likely to have a value around zero.

\section{Nonlinearity of Fungus-Fungus Combinations}

For each pair of fungal isolates ( $A$ and $B$ ), an analysis of variance (ANOVA) model of standardized growth index was constructed by including the presence/absence of isolate $A$, the presence/absence of isolate $B$, and the interaction term of the two (i.e., isolate $\mathrm{A} \times$ isolate $\mathrm{B}$ ) as explanatory variables. Then, across the 78 fungal pairs examined, $F$ values of the isolate $\mathrm{A} \times$ isolate $\mathrm{B}$ term were compared as indicators of how combinations of the two fungal isolates had "nonlinear" effects on plant performance traits. We then examined how the nonlinearity measures of fungal pairs are associated with the abovementioned index values representing deviations of observed dual-inoculation results from those expected as intermediate results of single inoculations $\left(\overline{D I_{A B}}\right)$.

All the calculations of the above indexes and statistical analyses were performed using the $\mathrm{R}$ base ver. 3.6.0 ( $\mathrm{R}$ Core Team, 2020).

\section{RESULTS}

\section{Germination Rates}

The gemination rates of Brassica plants varied within single inoculation treatments and within dual inoculation treatments (Supplementary Figure 2). Meanwhile, the rates were generally higher in dual inoculation treatments than in single inoculation treatments (Welch's test; $t=-3.97, \mathrm{df}=13.6, P=0.0015$ ).

\section{Plants' Growth Responses}

For all the three plant performance variables (shoot dry weight, the number of mature leaves, and SPAD), the single inoculation effects on Brassica plants differed significantly among the 13 fungal isolates examined (Table 2). For example, the mean standardized growth index for $V$. simplex Y34 and Alternaria sp. KYOCER00001239 were, on average, ca. seven-fold larger than the standard deviation of control sample's growth (i.e., $\overline{S G_{T}}>7$ ) in terms of shoot dry weight, indicating high growth-promoting effects of those fungi on Brassica plants (Figure 2A). In addition, C. chaetospira M4006, Trichoderma sp. KYOCER00000218, Curvularia sp. KYOCER00000077, Phoma sp. KYOCER00000052, and Stemphylium sp. KYOCER00000804 showed high plant growth promoting effects (Figure 2A). In contrast, C. tofieldiae MAFF 712334, Mucor sp. KYOCER00000113, Setosphaeria sp. KYOCER00000031, Fusarium sp. KYOCER00000983, and Tolypocladium sp. KYOCER00000289 displayed weak or almost neutral effects on plant growth and Aspergillus sp. KYOCER00000917 had negative impacts
TABLE 2 | Analysis of variance (ANOVA) results of single- and dual-inoculation experiments.

\begin{tabular}{lccc}
\hline ANOVA model & df & $\boldsymbol{F}$ & $\boldsymbol{P}$ \\
\hline Single inoculation (across $\mathbf{1 3}$ fungal isolates) & & & \\
Shoot dry weight & 12 & 41.6 & $<0.0001$ \\
Number of mature leaves & 12 & 127.7 & $<0.0001$ \\
Chlorophyll concentrations & 12 & 12.0 & $<0.0001$ \\
Dual inoculation (across 78 fungal pairs) & & & \\
Shoot dry weight & 77 & 25.7 & $<0.0001$ \\
Number of mature leaves & 77 & 23.1 & $<0.0001$ \\
Chlorophyll concentrations & 77 & 5.7 & $<0.0001$
\end{tabular}

For each of the three plant performance variables, an ANOVA model was constructed to examine the variation across single- or dual-inoculation treatments.

on the Brassica plants (Figure 2A). When the number of mature leaves was used as a metric of plant performance, Alternaria sp. KYOCER00001239 and Aspergillus sp. KYOCER00000917 turned out to have strongly positive and negative effects, respectively (Figure 2B). Meanwhile, the effects of other fungal isolates were moderately positive or neutral (Figure 2B).

In the dual inoculation experiments, the pair of the fungal isolates that exhibited the greatest effects in single inoculation treatments (i.e., $V$. simplex Y34 and Alternaria sp. KYOCER00001239) had relatively weak positive effects on Brassica growth in terms of shoot dry weight (Figure 2A). Instead, the highest plant-growth promoting effects were observed for the combination of $V$. simplex $\mathrm{Y} 34$ and Fusarium sp. KYOCER00000983, which had neutral effects on plants in the single inoculation (Figure 2A). Highly positive effects on plants (e.g., $\overline{S G_{T}}>5$ ) were observed, as well, in Curvularia-Fusarium, Cladophialophora-Alternaria, Colletotrichum-Cladophialophora, Aspergillus-Alternaria, and Cladophialophora-Veronaeopsis pairs and several other pairs including Curvularia sp. KYOCER00000077: for these pairs, at least one partner had neutral to weakly positive performance in single inoculation treatments (Figure 2A).

In contrast to those combinations with relatively high plantgrowth promoting effects (in the metrics of shoot dry weight and the number of mature leaves), Aspergillus sp. KYOCER00000917, which restricted plant growth in the single inoculation condition (Figures 2A,B), had negative impacts on plants in some of the 12 combinations with other fungal isolates (Figures $\mathbf{3 A}, \mathbf{B}$ ). However, their negative effects diminished in dual inoculations with some fungi such as Alternaria sp. KYOCER00001239 and Curvularia sp. KYOCER00000077 (Figures 3A,B). Results also showed that Phoma sp. KYOCER00000052, whose impacts on plants were positive in the single inoculation setting, inhibited plant growth in the presence of other fungi (Figures $3 \mathbf{A}, \mathbf{B}$ ).

When SAPD readings were used as metrics of plant performance, the Curvularia sp. KYOCER00000077 and Fusarium sp. KYOCER00000983 had relatively high positive effects on Brassica plants $\left(\overline{S G_{T}} \approx 2\right)$, while Setosphaeria sp. KYOCER00000031 and Aspergillus sp. KYOCER00000917 had negative impacts (Figure 2C). Note that chlorophyll 


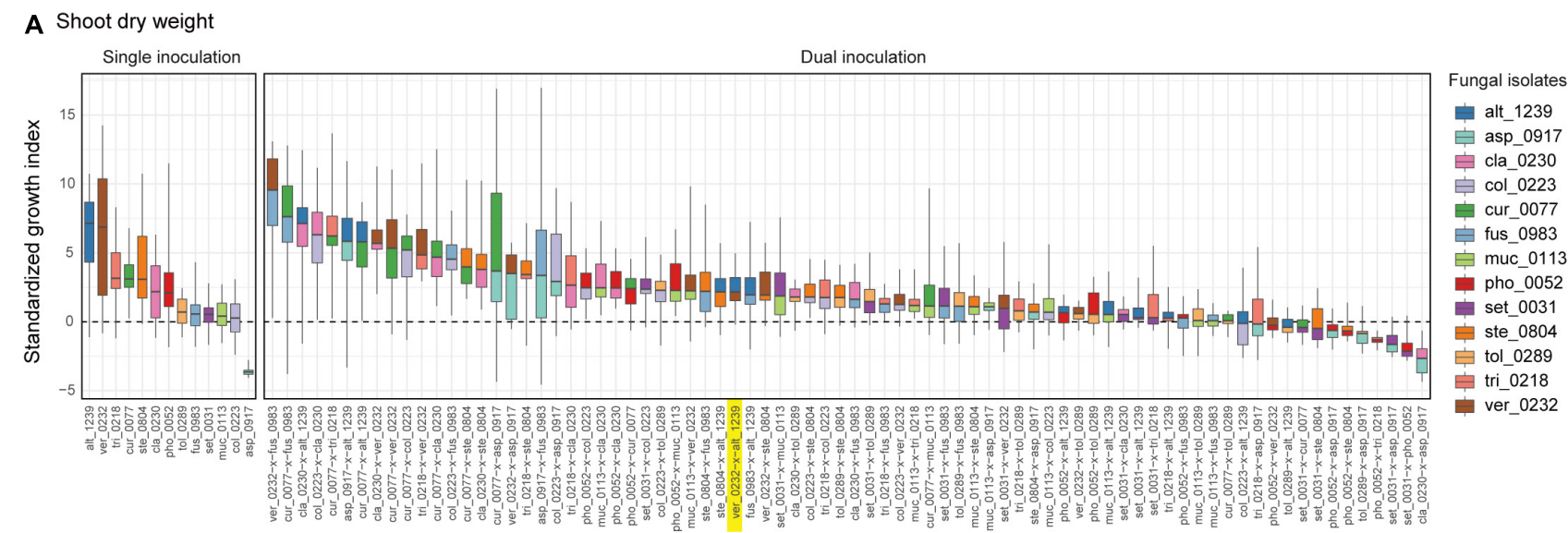

B Number of mature leaves

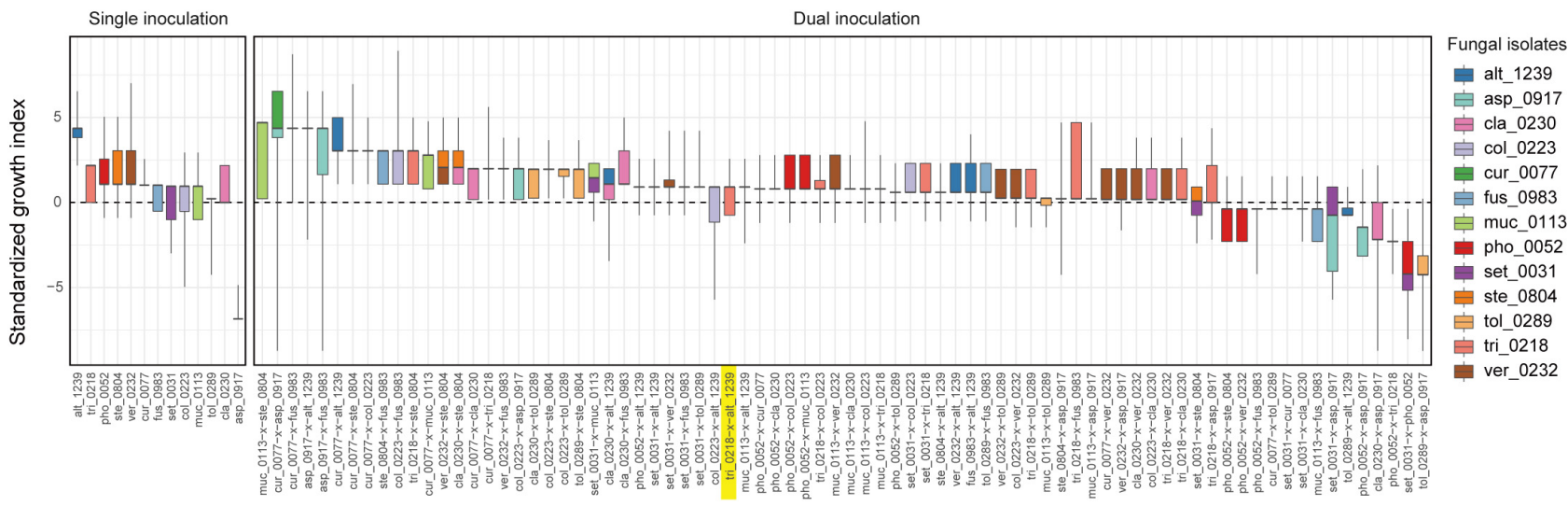

C Chlorophyll concentrations

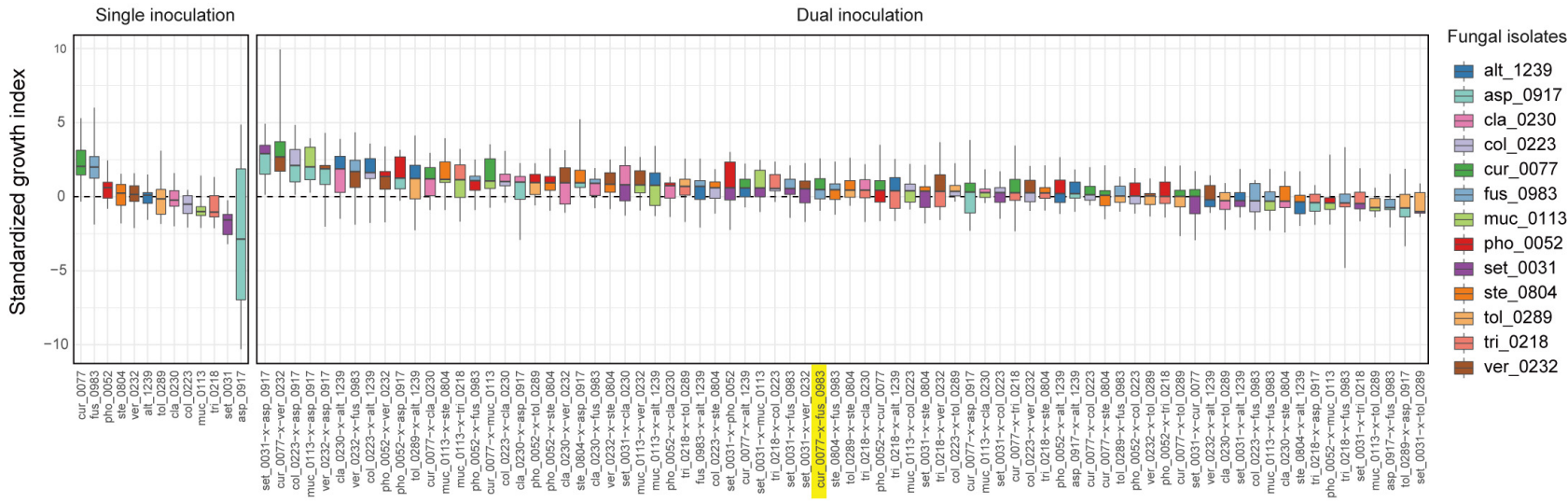

FIGURE 2 | Single- and dual-inoculation effects on Brassica plants. (A) Standardized growth index in terms of shoot dry weight. For respective single- and dual-inoculation experiments, $25 \%$ quantiles, medians, and $75 \%$ quantiles are displayed as boxes and the ranges from the maximum to minimum values are shown as bars. See Table $\mathbf{1}$ for the abbreviation of fungal isolates. The combination of the fungal species with the largest positive effects on Brassica plants in single inoculation experiments is highlighted. (B) Standardized growth index in terms of the number of mature leaves. (C) Standardized growth index in terms of chlorophyll concentrations.

concentrations were weakly correlated with shoot dry weight and the number of mature leaves (Supplementary Figure 3). In the dual inoculation experiments, some fungal pairs including Aspergillus sp. KYOCER00000917 had relatively high positive effects on Brassica plants (Figure 3C) despite negative impacts of the Aspergillus isolate in a single-inoculation 


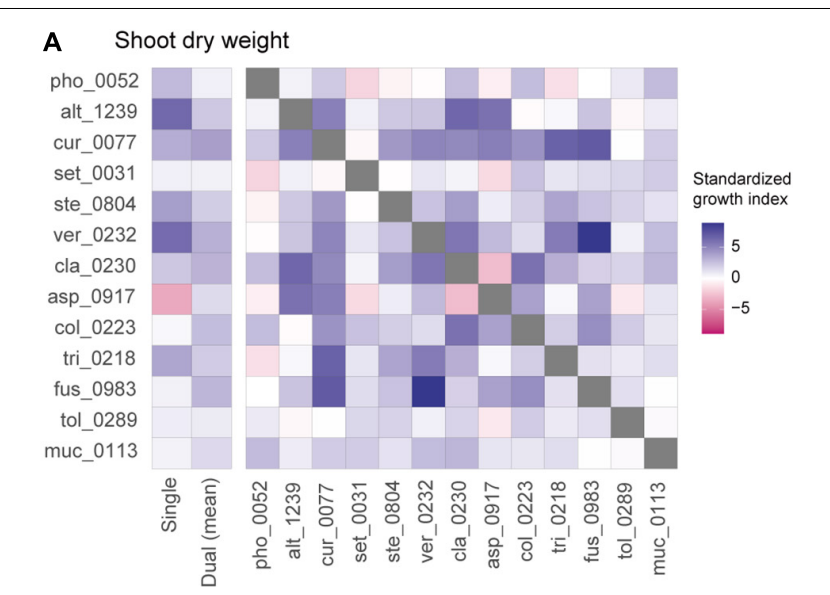

B Number of mature leaves

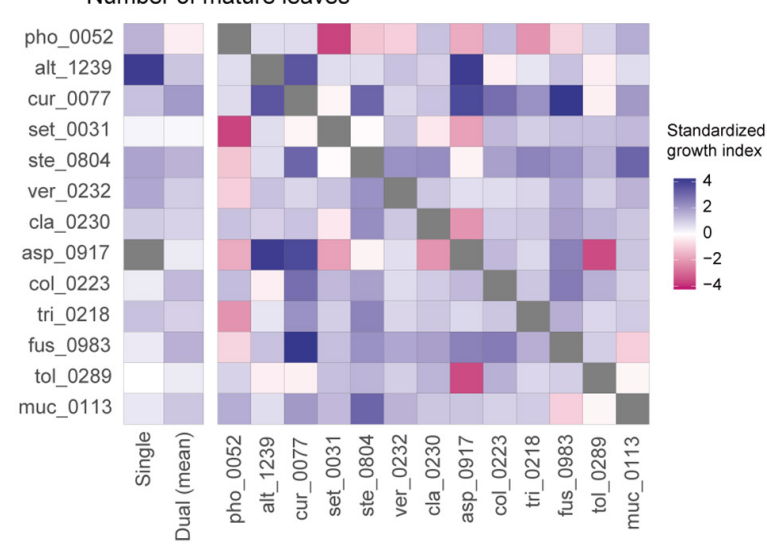

C Chlorophyll concentrations

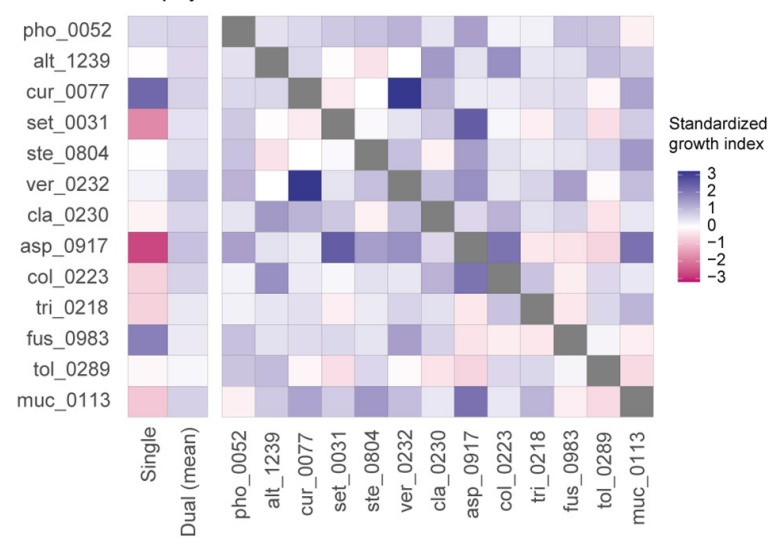

FIGURE 3 | Pairwise representation of dual inoculation results.

(A) Standardized growth index in terms of shoot dry weight for each pair of fungal isolates. Single-inoculation effects and mean effects across the dual inoculation assays are shown for each fungal isolate in the left.

(B) Standardized growth index in terms of the number of mature leaves.

(C) Standardized growth index in terms of chlorophyll concentrations.

condition (Figure 2C). The pair of Curvularia and Veronaeopsis moderately increased chlorophyll concentrations as well (Figure 3C). Meanwhile, chlorophyll concentrations did not differ greatly from the control for most fungal pairs (Figure 2C).

For all the three plant performance variables examined, standardized growth index values of single inoculation experiments were uncorrelated with those averaged across dual inoculations for respective fungi (shoot dry weight, $r=-0.09, P=0.78$; number of mature leaves $r=0.11, P=0.71$; SPAD, $r=-0.41, P=0.17$; Figure 3). In other words, fungi with more positive effects on plants in single-inoculation experiments did not increased plant performance more efficiently. The experimental results also indicated that some combinations of fungi exhibited higher impacts on Brassica performance than that observed in all the single-inoculation settings (Figures 2A-C).

\section{Synergistic and Offset Effects}

Among the 78 combinations of fungal isolates, strong synergistic effects $\left[\overline{S G_{A B}}>\max \left(\overline{S G_{A}}, \overline{S G_{B}}\right)\right]$ were observed in some pairs of fungi in terms of shoot dry weight (Figure 4A). The fungal combinations with the largest synergistic effects $\left(\overline{D M X_{A B}}\right)$ consisted of Curvularia sp. KYOCER00000077 and Fusarium sp. KYOCER00000983, each of which had weakly or moderately positive impacts on plant growth in single inoculations. Large synergistic effects were detected in other pairs of fungi including fungi with moderate or weakly positive effects on plants (e.g., Colletotrichum-Cladophialophora, Colletotrichum-Fusarium, and Veronaeopsis-Fusarium pairs; Figure 4A). Similarly, for the number of mature leaves, fungal pairs with large synergistic effects involved fungi with weakly positive or even negative effects in single inoculations (Figure 4B). In terms of chlorophyll concentrations, pairs of fungi with negative impacts on plants under single-inoculation conditions had large synergistic effects (Figure 4C).

In contrast to synergistic effects, offset effects $\left[\overline{S G_{A B}}<\min \left(\overline{S G_{A}}, \overline{S G_{B}}\right)\right]$ were evident especially in the fungal pairs including fungi that had highly positive impacts on plant performance traits under single-inoculation conditions (Figure 5). In particular, the pairs of fungi with the largest positive effects (i.e., the Veronaeopsis-Alternaria pair) showed large offset effects (Figure 5).

Across the 78 combinations of fungi, synergistic effects (i.e., $\overline{D M X_{A B}}$ ) decreased with increasing mean values of single inoculation effects of the target fungi (i.e., $\frac{\overline{S G_{A}}+\overline{S G_{B}}}{2}$ ) (Figures 6A-C). In other words, pairs of fungi that showed greater plant-performance increasing effects tended to have weaker synergistic effects. As expected by the trend in synergistic effects, offset effects were increased with increasing mean values of single inoculation effects of the target fungi (Figures 6D-F).

\section{Nonlinearity of Fungus-Fungus Combinations}

Deviations of observed dual-inoculation results from those expected as intermediate results of single inoculations $\left(\overline{D I_{A B}}\right)$ varied among fungal pairs (Figure 6). Higher absolute $\overline{D I_{A B}}$ values were indicative of nonlinearity in effects on plants for the particular fungus-fungus combinations as evaluated by a series of ANOVA models (Figure 7 and Supplementary Data 3). 

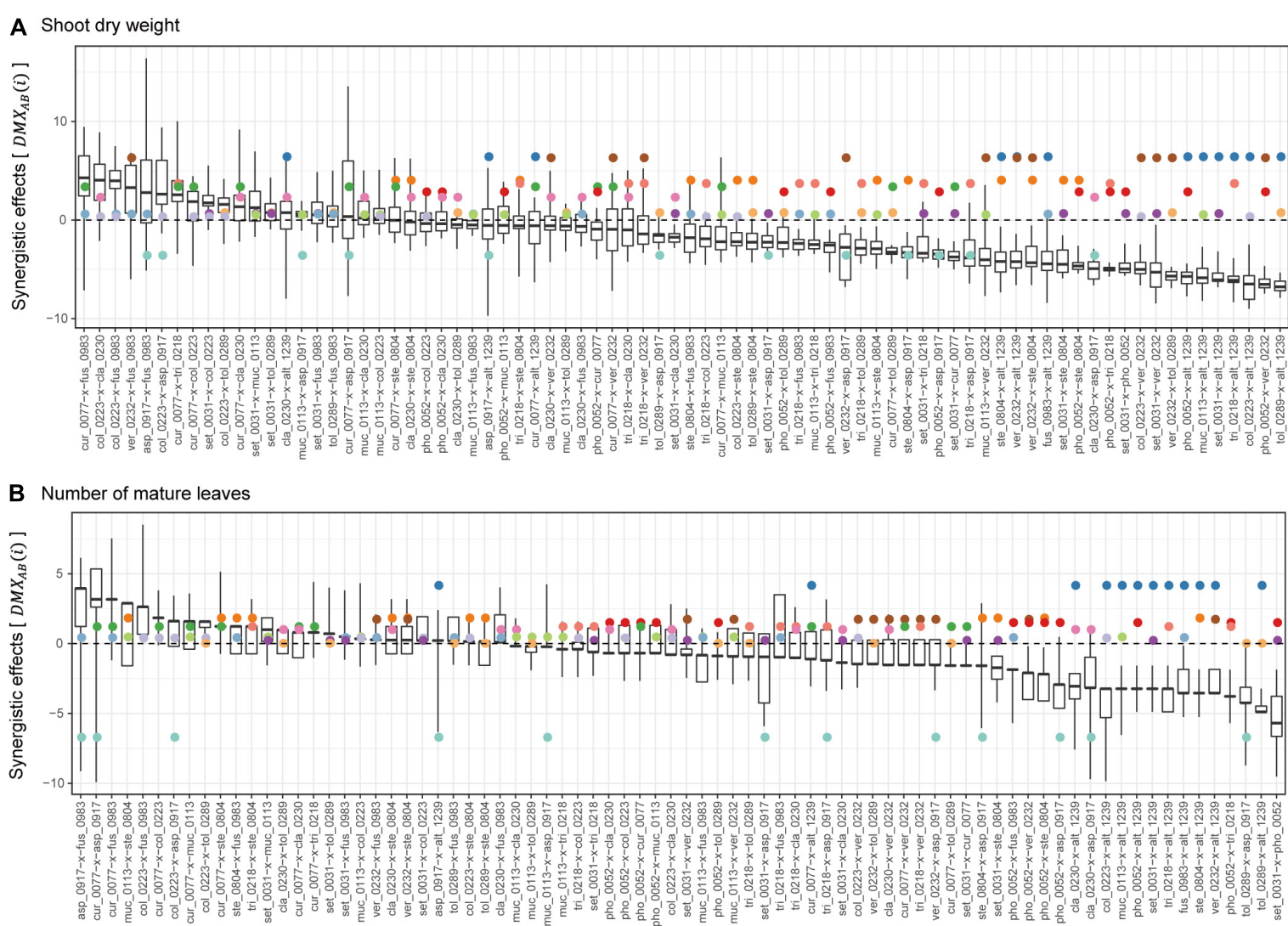

C Chlorophyll concentrations
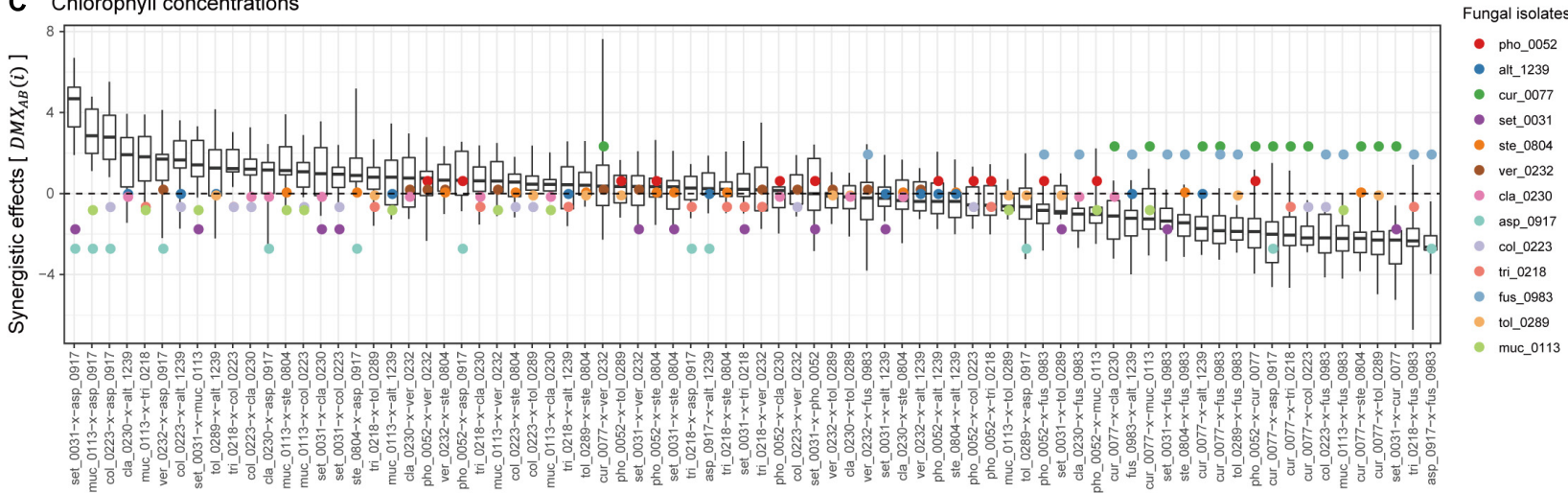

FIGURE 4 | Synergistic effects observed in dual-inoculation experiments. (A) Synergistic effect index in terms of shoot dry weight. The index representing deviation of dual-inoculation effects from the maximum effects in single inoculations are shown for each pair of fungal isolates. Circles represent single-inoculation effects of respective fungal isolates. (B) Synergistic effect index in terms of the number of mature leaves. (C) Synergistic effect index in terms of chlorophyll concentrations.

\section{DISCUSSION}

By using taxonomically diverse plant-associated fungi, we here evaluated plant-growth promoting effects of pairs of fungal isolates in light of those observed in single-isolate inoculation experiments. The 13 fungal isolates differed greatly in their independent effects on Brassica plants (Figures 2, 3), providing an ideal opportunity for examining how the ranking of plantgrowth promoting effects in single-inoculation contexts were related to that in multi-species (dual-inoculation) contexts
(Figures 4-6). Such information of synergistic and offset effects in the presence of multiple microbial species is indispensable for understanding to what extent we can predict functions of microbial communities (microbiomes) from the datasets of single-species/isolate screening.

A series of single- and dual-inoculation experiments indicated that greater performance of plants were potentially obtained in multi-species than in single-species contexts (Figure 2). This result, itself, is consistent with previous reports of enhanced plant growth by specific pairs of bacteria/fungi (Han and Lee, 2006; 

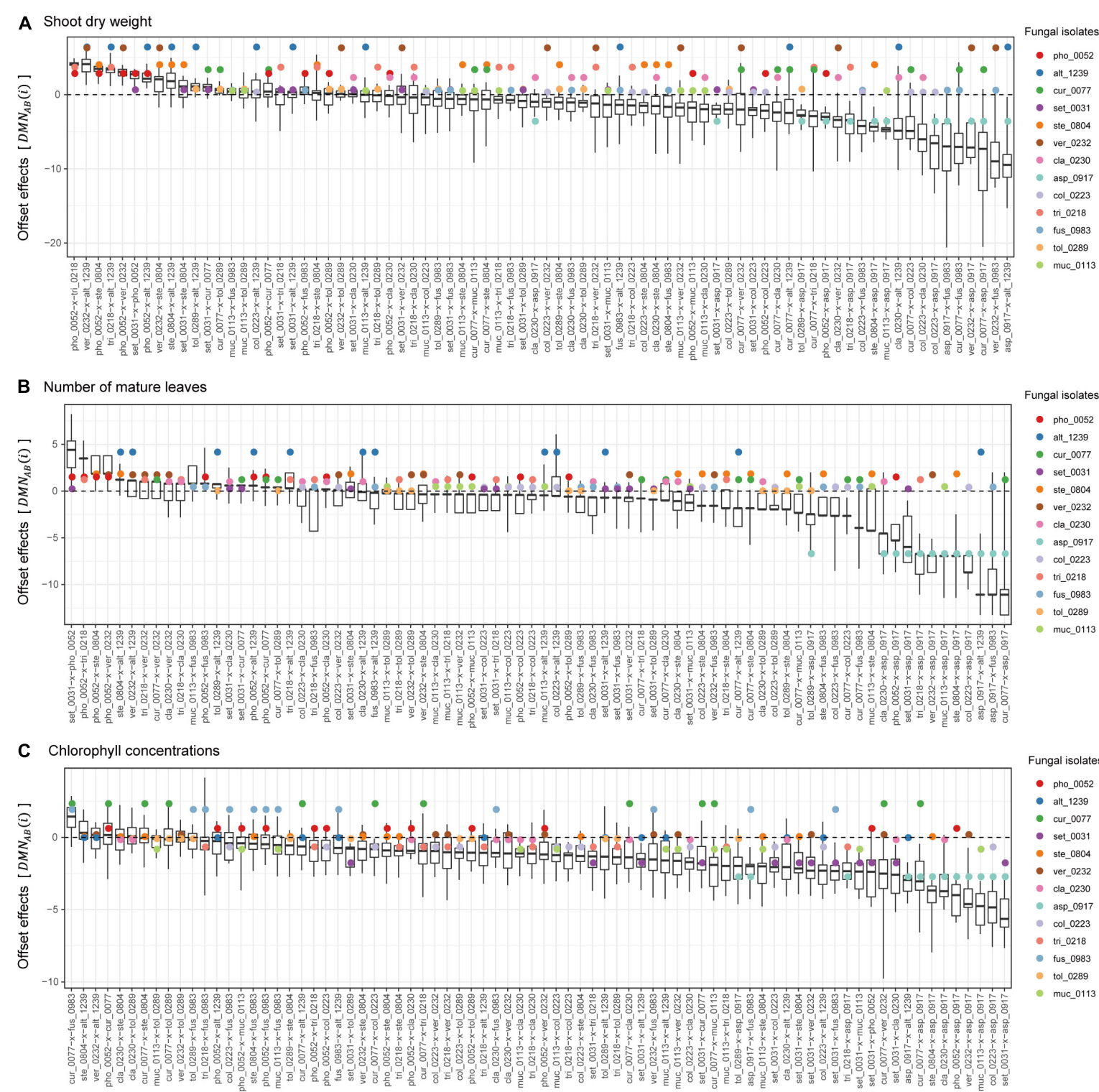

C Chlorophyll concentrations

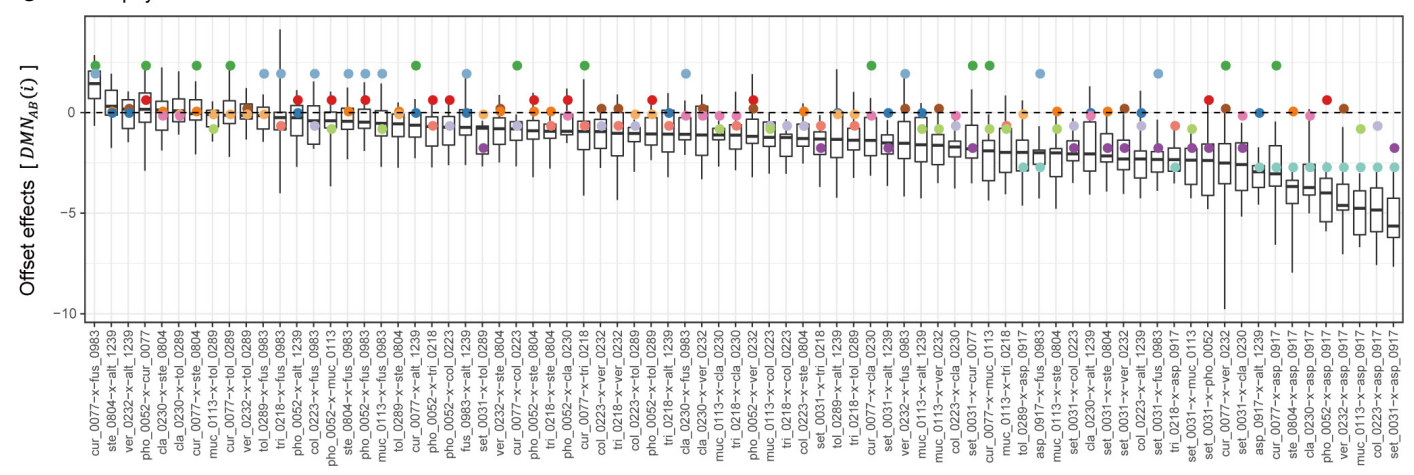

FIGURE 5 | Offset effects observed in dual-inoculation experiments. (A) Offset effect index in terms of shoot dry weight. The index representing deviation of dual-inoculation effects from the minimum effects in single inoculations are shown for each pair of fungal isolates. Circles represent single-inoculation effects of respective fungal isolates. (B) Offset effect index in terms of the number of mature leaves. (C) Offset effect index in terms of chlorophyll concentrations.

Wang et al., 2011; Ważny et al., 2018; He et al., 2020). Meanwhile, our experiments on 78 combinations of fungi systematically suggested that pairs of microbes, each of which had greatly positive impacts on plant growth in single inoculations, could show minor effects on plants under multi-species conditions. For example, the strategy of combining the two species/isolates with highest performance in the single inoculation experiments (i.e., $V$. simplex and Alternaria sp. KYOCER00001239) did not result in high plant-growth promoting effects (Figure 3): rather, offset effects were observed in those "top $\times$ top" pairs (Figures 4-6). Thus, biological functions at the community (microbiome) level may be rarely maximized by the "bottom-up" exploration of sets of microbes based solely on single-inoculation experiments.
Our experiments also suggested that pairs of microbes with subordinate performance in single inoculation assays could show largest growth-promoting effects on plants (Figure 2). This result suggests that single-species/isolate screening does not always provide sufficient information for predicting microbial performance at the multi-species level (Toju et al., 2018a). Interestingly, the fungal pairs with highest synergistic effects in our experiment involved fungi in the genera Fusarium and Curvularia (Figure 4A), which were often described as plant pathogenic taxa (Michielse and Rep, 2009; Ma et al., 2013; Manamgoda et al., 2015). Basically, physiological effects on plants vary remarkably among species/isolates within taxa as evidenced by the presence of Fusarium and Curvularia species enhancing 
A Shoot dry weight

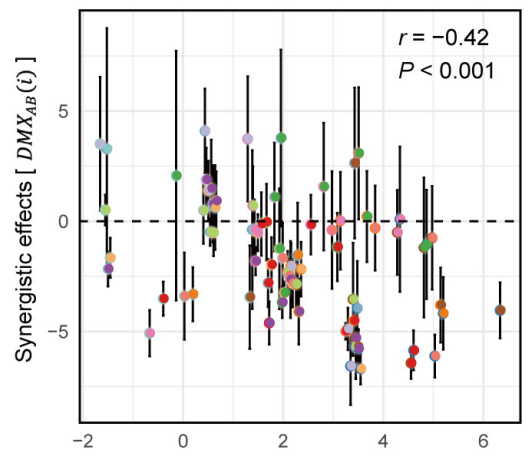

D Shoot dry weight

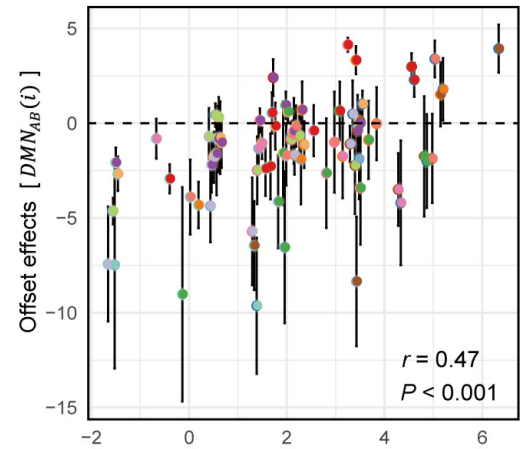

B Number of mature leaves

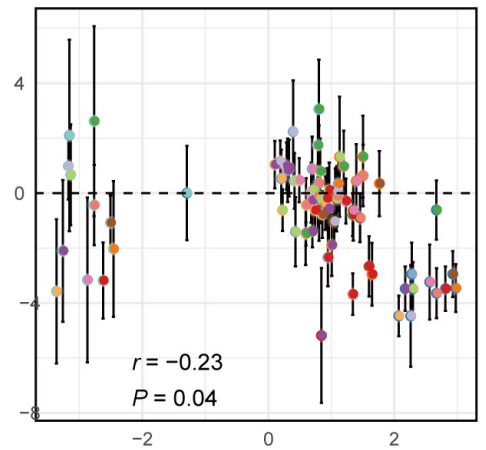

E Number of mature leaves

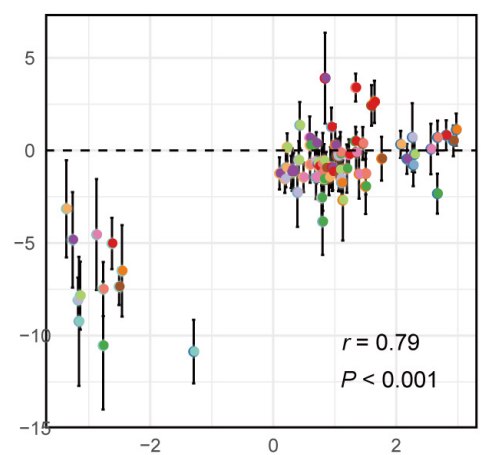

C Chlorophyll concentrations

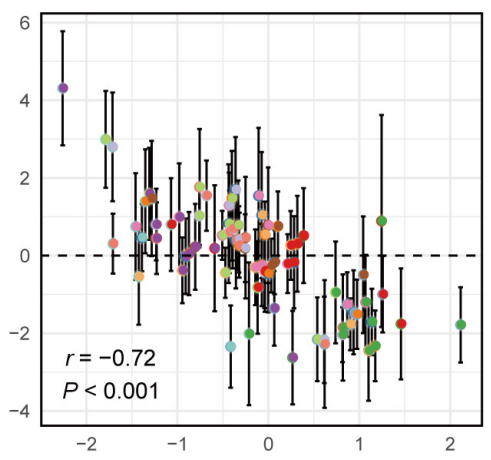

F Chlorophyll concentrations

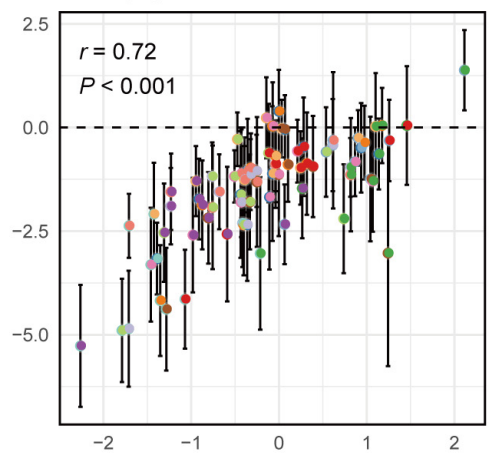

Mean of single inoculation effects $\frac{\overline{S G_{A}}+\overline{S G_{B}}}{2}$ )

FIGURE 6 | Relationship between single-inoculation effects and synergistic/offset effects. (A) Trends in synergistic effects in terms of shoot dry weight. For each pair of fungi, mean values of single inoculation effects of the target fungi (i.e., $\frac{\overline{S G_{A}}+\overline{S G_{B}}}{2}$ ) and index values of synergistic effects [i.e., $\left.D M X_{A B}(i)\right]$ are shown at the horizontal and vertical axes, respectively. Error bars represent standard deviations of synergistic effects. (B) Trends in synergistic effects in terms of the number of mature leaves. (C) Trends in synergistic effects in terms of chlorophyll concentrations. (D) Trends in synergistic effects in terms of shoot dry weight. For each pair of fungi, mean values of single inoculation effects of the target fungi (i.e., $\frac{\overline{S G_{A}}+\overline{S G_{B}}}{2}$ ) and index values of offset effects [i.e., $\left.D M N_{A B}(i)\right]$ are shown at the horizontal and vertical axes, respectively. (E) Trends in synergistic effects in terms of the number of mature leaves. (F) Trends in synergistic effects in terms of chlorophyll concentrations.

plant health and growth (Olivain et al., 2006; Nahalkova et al., 2008; Priyadharsini and Muthukumar, 2017). In fact, the Fusarium and Curvularia isolates examined in our study had positive effects on Brassica plants even in the single-inoculation assays (Figure 2). Moreover, the results of the dual inoculation experiments suggested that some fungi in these predominantly plant-pathogenic genera can have even greater positive effects on plants in combination with specific other fungi (Figures 2, 3). Our results on synergistic effects in multi-species contexts further illuminate the potential use of diverse endosphere/rhizosphere microbes whose biological functions have been underestimated in conventional screening of single inoculations.

The fact that microbial functions critically depend on combinations of microbial species/isolates highlight the importance of "bird's-eye" views of designing microbiomes. Given that microbial functions at the community (multispecies) levels are not the simple sums/averages of functions in single-species contexts (Figures 2, 7), research strategies taking into account not only each microbe's roles but also the nature of microbe-microbe interactions will provide platforms for optimization of microbiome functions (Agler et al., 2016; Toju et al., 2016; Banerjee et al., 2018). In this respect, interdisciplinary approaches integrating the observational, genomic, and metagenomic information of microbial functions (Bulgarelli et al., 2015; Levy et al., 2018; Ichihashi et al., 2020) with community ecological analyses of species interaction networks (Agler et al., 2016; van der Heijden and Hartmann, 2016; Toju et al., 2017) will help us explore highly functional and 


\section{A Shoot dry weight}

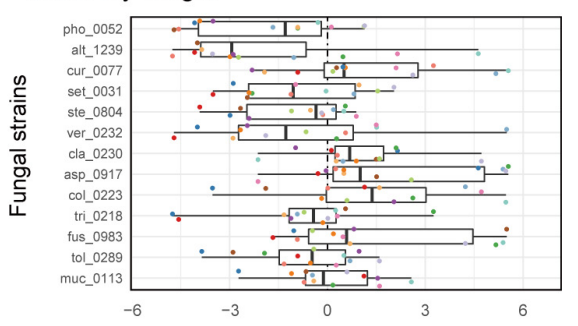

\section{B Number of mature leaves}
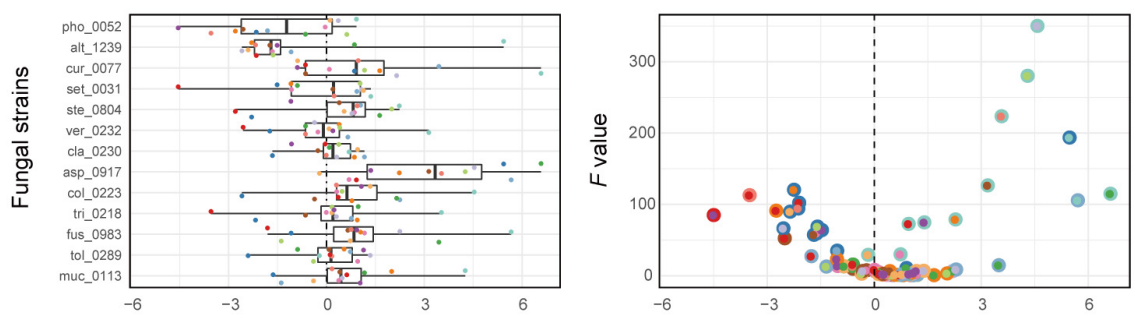

\section{Chlorophyll concentrations}

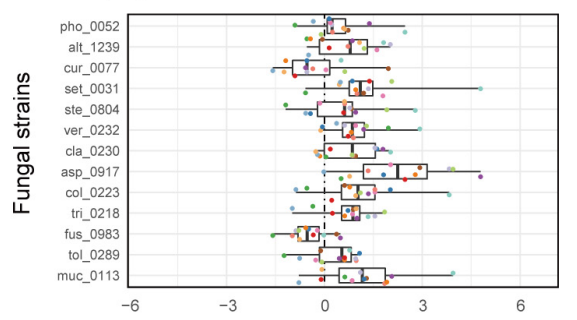

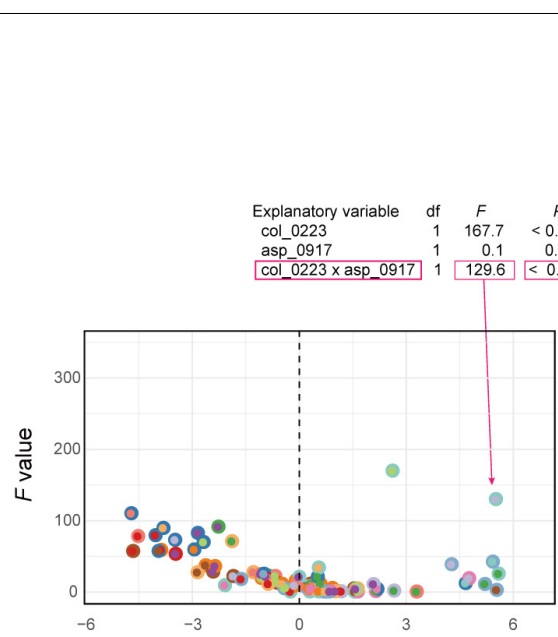

Fungal isolates

- pho_0052 asp_0917

- alt_1239 col_0223

- cur_0077 tri_0218

- set_0031 - fus_0983

- ste_0804 tol_0289

- ver_0232 muc_0113 - cla_0230

(
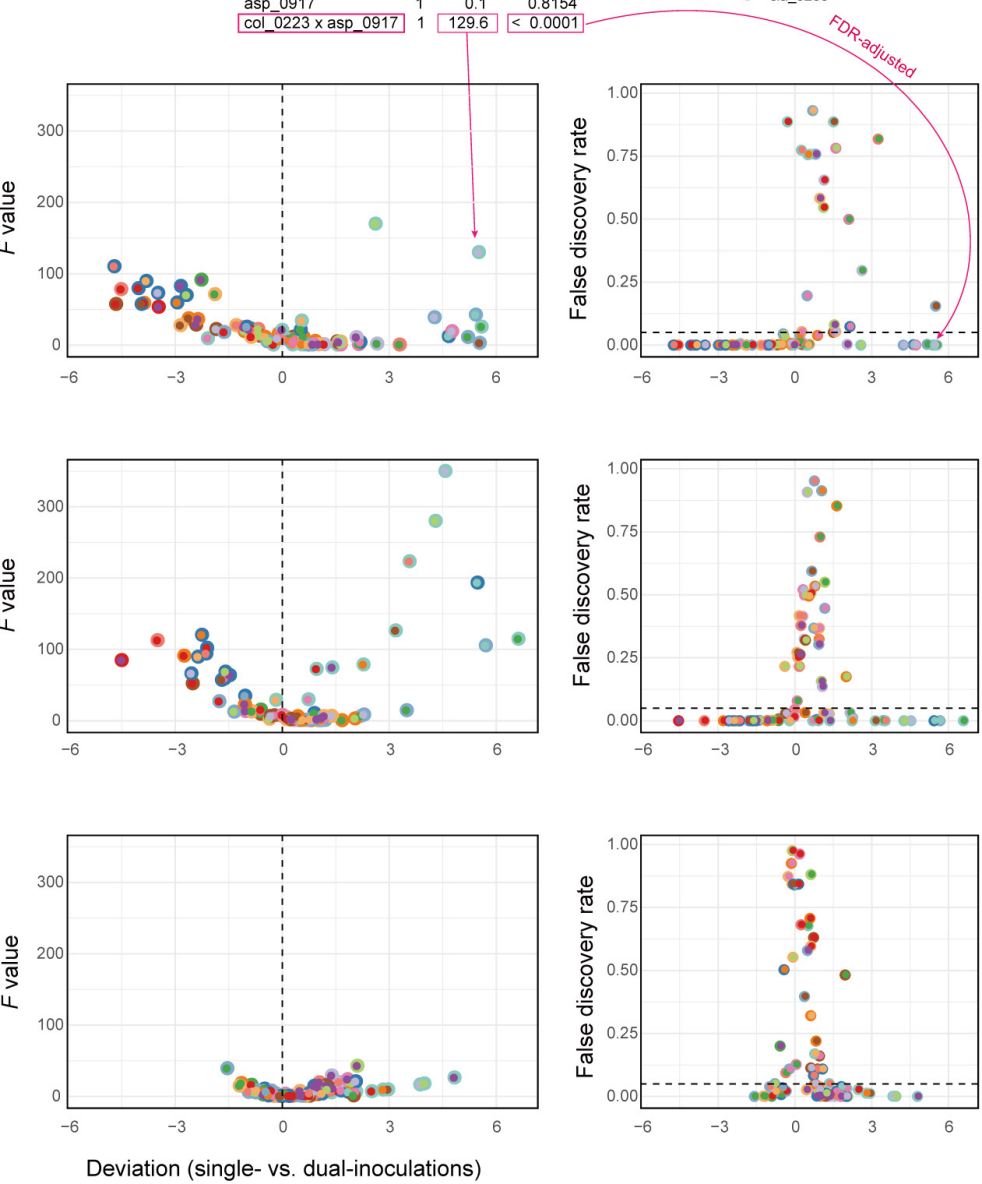

[DI $I_{A B}(i)$ ]

FIGURE 7 | Deviations of observed dual-inoculation results from those expected as intermediate results of single inoculations. (A) Deviation index for shoot dry weight. The index values representing deviations of dual-inoculation effects from intermediate effects in single inoculations $(\overline{D / A B}(i))$ are shown for each fungal isolate included in the target fungal pairs (left). For each fungal pair, $F$ values of the isolate $A \times$ isolate $B$ term in the ANOVA model (middle) and false discovery rate (FDR) values of the interaction term (right) are shown across the axis of the deviation index: FDR are calculated across the 78 fungal combinations examined. (B) Deviation index for the number of mature leaves. (C) Deviation index for chlorophyll concentrations.

stable microbial sets among numerous candidate combinations of species (Paredes et al., 2018; Saad et al., 2020; Toju et al., 2020). In other words, information of microbial functions in single-species contexts is utilized by being combined with insights into dynamics and processes within microbiomes.

While the experiments conducted in this study provided a unique opportunity for systematically evaluating synergistic/offset effects of microbes on plants, the obtained datasets should be interpreted with caution given the following limitations. First, physiological mechanisms by which the examined fungi affected plant growth were unexplored in the current study. Although detailed physiological and/or molecular biological investigations have been done for some of the fungal species used in this study [e.g., C. tofieldiae (Hiruma et al., 2016), $V$. simplex (Guo et al., 2018), and C. chaetospira (Harsonowati et al., 2020)], metabolites and genes involved in the plant-fungus interactions are unknown for the remaining species. For more mechanistic understanding of interactions involving plants and multiple microbial species, we need to perform transcriptomic analyses targeting plants' responses to each microbe as well as those comparing plants' gene expression patterns between singleand multiple-symbiont conditions. Comparative transcriptomic analyses across experiments with different environmental conditions (e.g., soil nutrient concentrations) will provide essential insights into microbial functions as well. Second, the inoculation test based on single plant species precluded us from 
understanding how general synergistic/offset effects existed in plant-fungal biome interactions. Although some of the fungal taxa used in this study have been reported to interact with multiple families of plants (Hermosa et al., 2012; Toju et al., 2018 b), impacts of endophytic/soil fungi on plants can vary depending on plant taxa and environmental conditions (Kiers et al., 2011; Pineda et al., 2013; Rudgers et al., 2020). Therefore, to gain more robust insights into synergistic/offset effects in interactions of plants and multiple microbial species/isolates, the reproducibility of the patterns observed in this study should be examined in inoculation experiments targeting diverse other plant species. Third, it is important to acknowledge that the complexity of the microbial sets examined in this study is minimal (i.e., two fungal species): different types of phenomena may be observed in combinations of three or more bacterial/fungal species (Durán et al., 2018; Paredes et al., 2018; Carlström et al., 2019; Wei et al., 2019). Moreover, it remains to be examined how we can increase microbial functions (e.g., host plant growth rates) by increasing the number of microbial species/isolates. The presence of microbial pairs outperforming single-microbe systems (Figure 2) leads to the working hypothesis that compatible sets of three or more microbial species yield greater functions than simpler communities by playing complementary roles. Meanwhile, it is expected that benefits of microbiomes do not increase linearly with increasing number of microbial species (i.e., saturating curves of benefits against increasing number of microbes) (van der Heijden et al., 1998), at least in terms of specific functions such as provisioning of soil phosphorus or blocking of soil pathogens.

We here showed that screening based on inoculations of single microbial species/isolates can result in the underestimation of the microbes that potentially have large plant-growth promoting effects in combinations with specific other microbes. Given that plants are inevitably associated with hundreds or more of microbial species in agricultural and natural ecosystems (Lundberg et al., 2012; Schlaeppi and Bulgarelli, 2015; van der Heijden and Hartmann, 2016; Thoms et al., 2021), such nonlinearity found in microbe-microbe associations deserve future intensive research. It may be important, for instance, to examine how antagonistic relationships between salicylicacid- and jasmonic-acid-related plant physiological responses (Niki et al., 1998), which are activated by different types of bacteria/fungi (Robert-Seilaniantz et al., 2011) [but see Betsuyaku et al. (2018)], can result in such nonlinear effects of multiple microbes on plant performance. Interdisciplinary

\section{REFERENCES}

Agler, M. T., Ruhe, J., Kroll, S., Morhenn, C., Kim, S. T., Weigel, D., et al. (2016). Microbial hub taxa link host and abiotic factors to plant microbiome variation. PLoS Biol. 14:e1002352. doi: 10.1371/journal.pbio.1002352

Ahmad, F., Ahmad, I., and Khan, M. S. (2008). Screening of free-living rhizospheric bacteria for their multiple plant growth promoting activities. Microbiol. Res. 163, 173-181. doi: 10.1016/j.micres.2006.04.001

Banerjee, S., Schlaeppi, K., and van der Heijden, M. G. A. (2018). Keystone taxa as drivers of microbiome structure and functioning. Nat. Rev. Microbiol. 16, 567-576. doi: 10.1038/s41579-018-0024-1 studies on relationships between microbiome compositions and their ecosystem-level functions are awaited toward the maximization of microbial functions for sustainable agriculture and ecosystem restoration.

\section{DATA AVAILABILITY STATEMENT}

The datasets presented in this study can be found in online repositories. The names of the repository/repositories and accession number(s) can be found below: DDBJ [accession: LC632034-LC632046]. Accession numbers for additional data relevant to this study can be found in the manuscript or Supplementary Material.

\section{AUTHOR CONTRIBUTIONS}

YH and HT designed the work. YH carried out the experiments. $\mathrm{YH}, \mathrm{HF}$, and HT analyzed the data. YH and HT wrote the manuscript based on discussion with $\mathrm{HF}, \mathrm{KH}$, and $\mathrm{KN}$. All authors contributed to the article and approved the submitted version.

\section{FUNDING}

This work was financially supported in part by Precursory Research for Embryonic Science and Technology (PRESTO) of Japan Science and Technology Agency (JST; JPMJPR16Q6 to HT), the Human Frontier Science Program (HFSP; RGP0029/2019 to HT), and the New Energy and Industrial Technology Development Organization (NEDO; JPNP18016 to HT).

\section{ACKNOWLEDGMENTS}

We thank reviewers for constructive comments on the manuscript.

\section{SUPPLEMENTARY MATERIAL}

The Supplementary Material for this article can be found online at: https://www.frontiersin.org/articles/10.3389/fmicb. 2021.713180/full\#supplementary-material

Betsuyaku, S., Katou, S., Takebayashi, Y., Sakakibara, H., Nomura, N., and Fukuda, H. (2018). Salicylic acid and jasmonic acid pathways are activated in spatially different domains around the infection site during effector-triggered immunity in Arabidopsis thaliana. Plant Cell Physiol. 59, 8-16. doi: 10.1093/pcp/pcx181

Bhattacharyya, P. N., and Jha, D. K. (2012). Plant growth-promoting rhizobacteria (PGPR): emergence in agriculture. World J. Microbiol. Biotechnol. 28, 13271350. doi: 10.1007/s11274-011-0979-9

Bulgarelli, D., Garrido-Oter, R., Münch, P. C., Weiman, A., Dröge, J., Pan, Y., et al. (2015). Structure and function of the bacterial root microbiota in wild and domesticated barley. Cell Host Microbe 17, 392-403. doi: 10.1016/j.chom. 2015.01.011 
Bulgarelli, D., Schlaeppi, K., Spaepen, S., van Themaat, E. V. L., and Schulze-Lefert, P. (2013). Structure and functions of the bacterial microbiota of plants. Ann. Rev. Plant Biol. 64, 807-838. doi: 10.1146/annurev-arplant-050312-120106

Busby, P. E., Soman, C., Wagner, M. R., Friesen, M. L., Kremer, J., Bennett, A., et al. (2017). Research priorities for harnessing plant microbiomes in sustainable agriculture. PLoS Biol. 15:e2001793. doi: 10.1371/journal.pbio.2001793

Carlström, C. I., Field, C. M., Bortfeld-Miller, M., Müller, B., Sunagawa, S., and Vorholt, J. A. (2019). Synthetic microbiota reveal priority effects and keystone strains in the Arabidopsis phyllosphere. Nat. Ecol. Evol. 3, 1445-1454. doi: 10. 1038/s41559-019-0994-z

Castrillo, G., Teixeira, P. J., Paredes, S. H., Law, T. F., de Lorenzo, L., Feltcher, M. E., et al. (2017). Root microbiota drive direct integration of phosphate stress and immunity. Nature 543, 513-518. doi: 10.1038/nature21417

Chang, S. X., and Robison, D. J. (2003). Nondestructive and rapid estimation of hardwood foliar nitrogen status using the SPAD-502 chlorophyll meter. Forest Ecol. Manag. 181, 331-338. doi: 10.1016/S0378-1127(03)00004-5

Dai, C. C., Yu, B. Y., and Li, X. (2008). Screening of endophytic fungi that promote the growth of Euphorbia pekinensis. Afr. J. Biotechnol. 7, 3505-3510. doi: 10.4314/ajb.v7i19.59361

Durán, P., Thiergart, T., Garrido-Oter, R., Agler, M., Kemen, E., Schulze-Lefert, P., et al. (2018). Microbial interkingdom interactions in roots promote Arabidopsis survival. Cell 175, 973-983.e14. doi: 10.1016/j.cell.2018.10.020

Esfahani, M., Abbasi, H. R. A., Rabiei, B., and Kavousi, M. (2008). Improvement of nitrogen management in rice paddy fields using chlorophyll meter (SPAD). Paddy Water Environ. 6, 181-188. doi: 10.1007/s10333-007-0094-6

Finkel, O. M., Salas-González, I., Castrillo, G., Conway, J. M., Law, T. F., Teixeira, P. J., et al. (2020). A single bacterial genus maintains root growth in a complex microbiome. Nature 587, 103-108. doi: 10.1038/s41586-020-2778-7

Gu, S., Wei, Z., Shao, Z., Friman, V. P., Cao, K., Yang, T., et al. (2020). Competition for iron drives phytopathogen control by natural rhizosphere microbiomes. Nat. Microbiol. 5, 1002-1010. doi: 10.1038/s41564-020-0719-8

Guo, Y., Matsuoka, Y., Nishizawa, T., Ohta, H., and Narisawa, K. (2018). Effects of rhizobium species living with the dark septate endophytic fungus veronaeopsis simplex on organic substrate utilization by the host. Microb. Environ. 33, 102-106. doi: 10.1264/jsme2.ME17144

Han, H. S., and Lee, K. D. (2006). Effect of co-inoculation with phosphate and potassium solubilizing bacteria on mineral uptake and growth of pepper and cucumber. Plant Soil Environ. 52, 130-136.

Harbort, C. J., Hashimoto, M., Inoue, H., Niu, Y., Guan, R., Rombolà, A. D., et al. (2020). Root-secreted coumarins and the microbiota interact to improve iron nutrition in Arabidopsis. Cell Host Microbe 28, 825-837. doi: 10.1016/j.chom. 2020.09.006

Harsonowati, W., Marian, M., Surono, M., and Narisawa, K. (2020). The effectiveness of a dark septate endophytic fungus, Cladophialophora chaetospira SK51, to mitigate strawberry Fusarium wilt disease and with growth promotion activities. Front. Microbiol. 11:585. doi: 10.3389/fmicb.2020.00585

He, C., Wang, W., and Hou, J. (2020). Plant performance of enhancing licorice with dual inoculating dark septate endophytes and Trichoderma viride mediated via effects on root development. BMC Plant Biol. 20:325. doi: 10.1186/s12870-02002535-9

Helfrich, E. J. N., Vogel, C. M., Ueoka, R., Schäfer, M., Ryffel, F., Müller, D. B., et al. (2018). Bipartite interactions, antibiotic production and biosynthetic potential of the Arabidopsis leaf microbiome. Nat. Microbiol. 3, 909-919. doi: 10.1038/ s41564-018-0200-0

Hermosa, R., Viterbo, A., Chet, I., and Monte, E. (2012). Plant-beneficial effects of Trichoderma and of its genes. Microbiology 158, 17-25. doi: 10.1099/mic.0. 052274-0

Hiruma, K., Gerlach, N., Sacristán, S., Nakano, R. T., Hacquard, S., Kracher, B., et al. (2016). Root endophyte Colletotrichum tofieldiae confers plant fitness benefits that are phosphate status dependent. Cell 165, 464-474. doi: 10.1016/j.cell.2016. 02.028

Hiruma, K., Kobae, Y., and Toju, H. (2018). Beneficial associations between Brassicaceae plants and fungal endophytes under nutrient-limiting conditions: evolutionary origins and host - symbiont molecular mechanisms. Curr. Opin. Plant Biol. 44, 145-154. doi: 10.1016/j.pbi.2018.04.009

Ichihashi, Y., Ichihashi, Y., Date, Y., Date, Y., Shino, A., Shimizu, T., et al. (2020). Multi-omics analysis on an agroecosystem reveals the significant role of organic nitrogen to increase agricultural crop yield. Proc. Natl. Acad. Sci. U.S.A. 117, 14552-14560. doi: 10.1073/pnas.1917259117
Jansa, J., Forczek, S. T., Rozmoš, M., Püschel, D., Bukovská, P., and Hršelová, H. (2019). Arbuscular mycorrhiza and soil organic nitrogen: network of players and interactions. Chem. Biol. Technol. Agric. 6:10. doi: 10.1186/s40538-019-0 $147-2$

Kennedy, P. G., Peay, K. G., and Bruns, T. D. (2009). Root tip competition among ectomycorrhizal fungi: are priority effects a rule or an exception? Ecology 90, 2098-2107.

Khastini, R. O., Ohta, H., and Narisawa, K. (2012). The role of a dark septate endophytic fungus, veronaeopsis simplex Y34, in fusarium disease suppression in Chinese cabbage. J. Microbiol. 50, 618-624. doi: 10.1007/s12275-012-2105-6

Kiers, E. T., Duhamel, M., Beesetty, Y., Mensah, J. A., Franken, O., Verbruggen, E., et al. (2011). Reciprocal rewards stabilize cooperation in the mycorrhizal symbiosis. Science 333, 880-882. doi: 10.1126/science.1208473

Levy, A., Salas Gonzalez, I., Mittelviefhaus, M., Clingenpeel, S., Herrera Paredes, S., Miao, J., et al. (2018). Genomic features of bacterial adaptation to plants. Nat. Genet. 50, 138-150. doi: 10.1038/s41588-017-0012-9

Lugtenberg, B., and Kamilova, F. (2009). Plant-growth-promoting rhizobacteria. Ann. Rev. Microbiol. 63, 541-556. doi: 10.1146/annurev.micro.62.081307. 162918

Lundberg, D. S., Lebeis, S. L., Paredes, S. H., Yourstone, S., Gehring, J., Malfatti, S., et al. (2012). Defining the core Arabidopsis thaliana root microbiome. Nature 488, 86-90. doi: 10.1038/nature 11237

Ma, L. J., Geiser, D. M., Proctor, R. H., Rooney, A. P., O’Donnell, K., Trail, F., et al. (2013). Fusarium pathogenomics. Ann. Rev. Microbiol. 67, 399-416. doi: 10.1146/annurev-micro-092412-155650

Manamgoda, D. S., Rossman, A. Y., Castlebury, L. A., Chukeatirote, E., and Hyde, K. D. (2015). A taxonomic and phylogenetic re-appraisal of the genus Curvularia (Pleosporaceae): human and plant pathogens. Phytotaxa 212, 175198. doi: 10.11646/phytotaxa.212.3.1

Michielse, C. B., and Rep, M. (2009). Pathogen profile update: Fusarium oxysporum. Mol. Plant Pathol. 10, 311-324. doi: 10.1111/j.1364-3703.2009. 00538.x

Nahalkova, J., Fatehi, J., Olivain, C., and Alabouvette, C. (2008). Tomato root colonization by fluorescent-tagged pathogenic and protective strains of Fusarium oxysporum in hydroponic culture differs from root colonization in soil. FEMS Microbiol. Lett. 286, 152-157. doi: 10.1111/j.1574-6968.2008.01 241.x

Nara, K. (2006). Ectomycorrhizal networks and seedling establishment during early primary succession. New Phytol. 169, 169-178. doi: 10.1111/j.1469-8137.2005. 01545.x

Narisawa, K., Usuki, F., and Hashiba, T. (2004). Control of verticillium yellows in Chinese cabbage by the dark septate endophytic fungus LtVB3. Phytopathology 94, 412-418. doi: 10.1094/PHYTO.2004.94.5.412

Nelson, J. M., Hauser, D. A., Hinson, R., and Shaw, A. J. (2018). A novel experimental system using the liverwort Marchantia polymorpha and its fungal endophytes reveals diverse and context-dependent effects. New Phytol. 218, 1217-1232. doi: 10.1111/nph.15012

Netto, A. T., Campostrini, E., de Oliveira, J. G., and Bressan-Smith, R. E. (2005). Photosynthetic pigments, nitrogen, chlorophyll a fluorescence and SPAD-502 readings in coffee leaves. Sci. Hortic. 104, 199-209. doi: 10.1016/j.scienta.2004. 08.013

Newsham, K. K. (2011). A meta-analysis of plant responses to dark septate root endophytes. New Phytol. 190, 783-793. doi: 10.1111/j.1469-8137.2010.03611.x

Nguyen, N. H., Song, Z., Bates, S. T., Branco, S., Tedersoo, L., Menke, J., et al. (2016). FUNGuild: an open annotation tool for parsing fungal community datasets by ecological guild. Fungal Ecol. 20, 241-248. doi: 10.1016/j.funeco. 2015.06.006

Niki, T., Mitsuhara, I., Seo, S., Ohtsubo, N., and Ohashi, Y. (1998). Antagonistic effect of salicylic acid and jasmonic acid on the expression of pathogenesisrelated (PR) protein genes in wounded mature tobacco leaves. Plant Cell Physiol. 39, 500-507. doi: 10.1093/oxfordjournals.pcp.a029397

Olivain, C., Humbert, C., Nahalkova, J., Fatehi, J., L'Haridon, F., and Alabouvette, C. (2006). Colonization of tomato root by pathogenic and nonpathogenic Fusarium oxysporum strains inoculated together and separately into the soil. Appl. Environ. Microbiol. 72, 1523-1531. doi: 10.1128/AEM.72.2.1523-1531. 2006

Paredes, H. S., Gao, T., Law, T. F., Finkel, O. M., Mucyn, T., Teixeira, P. J. P. L., et al. (2018). Design of synthetic bacterial communities for predictable plant phenotypes. PLoS Biol. 16:e2003962. doi: 10.1371/journal.pbio.2003962 
Peay, K. G., Kennedy, P. G., and Talbot, J. M. (2016). Dimensions of biodiversity in the Earth mycobiome. Nat. Rev. Microbiol. 14, 434-447. doi: 10.1038/nrmicro. 2016.59

Pieterse, C. M. J., Zamioudis, C., Berendsen, R. L., Weller, D. M., van Wees, S. C. M., and Bakker, P. A. H. M. (2014). Induced systemic resistance by beneficial microbes. Ann. Rev. Phytopathol. 52, 347-375. doi: 10.1146/annurev-phyto082712-102340

Pineda, A., Dicke, M., Pieterse, C. M. J., and Pozo, M. J. (2013). Beneficial microbes in a changing environment: are they always helping plants to deal with insects? Funct. Ecol. 27, 574-586. doi: 10.1111/1365-2435.12050

Priyadharsini, P., and Muthukumar, T. (2017). The root endophytic fungus Curvularia geniculata from Parthenium hysterophorus roots improves plant growth through phosphate solubilization and phytohormone production. Fungal Ecol. 27, 69-77. doi: 10.1016/j.funeco.2017.02.007

R Core Team. (2020). R: A Language and Environment for Statistical Computing. Vienna: R Foundation for Statistical Computing.

Radhakrishnan, R., Khan, A. L., Kang, S. M., and Lee, I. J. (2015). A comparative study of phosphate solubilization and the host plant growth promotion ability of Fusarium verticillioides RK01 and Humicola sp. KNU01 under salt stress. Ann. Microbiol. 65, 585-593. doi: 10.1007/s13213-014-0894-z

Remy, W., Taylor, T. N., Hass, H., and Kerp, H. (1994). Four hundred-millionyear-old vesicular arbuscular mycorrhizae. Proc. Nat. Acad. Sci. U.S.A. 91, 11841-11843. doi: 10.1073/pnas.91.25.11841

Richardson, A. E., Barea, J. M., McNeill, A. M., and Prigent-Combaret, C. (2009). Acquisition of phosphorus and nitrogen in the rhizosphere and plant growth promotion by microorganisms. Plant Soil 321, 305-339. doi: 10.1007/s11104009-9895-2

Robert-Seilaniantz, A., Grant, M., and Jones, J. D. G. (2011). Hormone crosstalk in plant disease and defense: more than just JASMONATE-SALICYLATE antagonism. Ann. Rev. Phytopathol. 49, 317-343. doi: 10.1146/annurev-phyto073009-114447

Rudgers, J. A., Afkhami, M. E., Bell-Dereske, L., Chung, Y. A., Crawford, K. M., Kivlin, S. N., et al. (2020). Climate disruption of plant-microbe interactions. Ann. Rev. Ecol. Evol. Syst. 51, 561-586. doi: 10.1146/annurev-ecolsys-011720090819

Saad, M. M., Eida, A. A., and Hirt, H. (2020). Tailoring plant-associated microbial inoculants in agriculture: a roadmap for successful application. J. Exp. Bot. 71, 3878-3901. doi: 10.1093/jxb/eraa111

Schlaeppi, K., and Bulgarelli, D. (2015). The plant microbiome at work. Mol. Plant Microbe Interact. 212, 212-217. doi: 10.1094/MPMI-10-14-0334-FI

Taurian, T., Anzuay, M. S., Angelini, J. G., Tonelli, M. L., Ludueña, L., Pena, D., et al. (2010). Phosphate-solubilizing peanut associated bacteria: screening for plant growth-promoting activities. Plant Soil 329, 421-431. doi: 10.1007/ s11104-009-0168-x

Taylor, T. N., Remy, W., Haas, H., and Kerp, H. (1995). Fossil arbuscular mycorrhizae from the early Devonian. Mycologia 87, 560-573. doi: 10.2307/ 3760776

Tedersoo, L., May, T. W., and Smith, M. E. (2010). Ectomycorrhizal lifestyle in fungi: global diversity, distribution, and evolution of phylogenetic lineages. Mycorrhiza 20, 217-263. doi: 10.1007/s00572-009-0274-x

Thoms, D., Liang, Y., and Haney, C. H. (2021). Maintaining symbiotic homeostasis: how do plants engage with beneficial microorganisms while at the same time restricting pathogens? Mol. Plant Microbe Interact. 34, 462-469. doi: 10.1094/ mpmi-11-20-0318-fi

Toju, H., Abe, M. S., Ishii, C., Hori, Y., Fujita, H., and Fukuda, S. (2020). Scoring species for synthetic community design: network analyses of functional core microbiomes. Front. Microbiol. 11:1361. doi: 10.3389/fmicb.20 20.01361

Toju, H., Peay, K. G., Yamamichi, M., Narisawa, K., Hiruma, K., Naito, K., et al. (2018a). Core microbiomes for sustainable agroecosystems. Nat. Plants 4, 247-257. doi: 10.1038/s41477-018-0139-4

Toju, H., Tanabe, A. S., and Sato, H. (2018b). Network hubs in root-associated fungal metacommunities. Microbiome 6:116. doi: 10.1101/270371

Toju, H., Yamamichi, M., Guimarães, P. R. Jr., Olesen, J. M., Mougi, A., Yoshida, T., et al. (2017). Species-rich networks and eco-evolutionary synthesis at the metacommunity level. Nat. Ecol. Evol. 1:54. doi: 10.1038/s41559-016-0024
Toju, H., Yamamoto, S., Tanabe, A. S., Hayakawa, T., and Ishii, H. S. (2016). Network modules and hubs in plant-root fungal biomes. J. R. Soc. Interf. 13:20151097. doi: 10.1098/rsif.2015.1097

Trivedi, P., Leach, J. E., Tringe, S. G., Sa, T., and Singh, B. K. (2020). Plantmicrobiome interactions: from community assembly to plant health. Nat. Rev. Microbiol. 18, 607-621. doi: 10.1038/s41579-020-0412-1

Tsolakidou, M. D., Stringlis, I. A., Fanega-Sleziak, N., Papageorgiou, S., Tsalakou, A., and Pantelides, I. S. (2019). Rhizosphere-enriched microbes as a pool to design synthetic communities for reproducible beneficial outputs. FEMS Microbiol. Ecol. 95:fiz138. doi: 10.1093/femsec/fiz138

Usuki, F., and Narisawa, K. (2007). A mutualistic symbiosis between a dark septate endophytic fungus, Heteroconium chaetospira, and a nonmycorrhizal plant, Chinese cabbage. Mycologia 99, 175-184. doi: 10.3852/mycologia.99.2.175

van der Heijden, M. G. A., and Hartmann, M. (2016). Networking in the plant microbiome. PLoS Biol. 14:e1002378. doi: 10.1371/journal.pbio.1002378

van der Heijden, M. G. A., Klironomos, J. N., Ursic, M., Moutoglis, P., StreitwolfEngel, R., Boller, T., et al. (1998). Mycorrhizal fungal diversity determines plant biodiversity, ecosystem variability and productivity. Nature 396, 69-72. doi: $10.1038 / 23932$

van Wees, S. C., van der Ent, S., and Pieterse, C. M. (2008). Plant immune responses triggered by beneficial microbes. Curr. Opin. Plant Biol. 11, 443-448. doi: 10.1016/j.pbi.2008.05.005

Vinale, F., Sivasithamparam, K., Ghisalberti, E. L., Marra, R., Woo, S. L., and Lorito, M. (2008). Trichoderma-plant-pathogen interactions. Soil Biol. Biochem. 40, 1-10. doi: 10.1016/j.soilbio.2007.07.002

Vorholt, J. A., Vogel, C., Carlström, C. I., and Müller, D. B. (2017). Establishing causality: opportunities of dynthetic vommunities for plant microbiome tesearch. Cell Host Microbe 22, 142-155. doi: 10.1016/j.chom.2017.07.004

Wagg, C., Schlaeppi, K., Banerjee, S., Kuramae, E. E., and van der Heijden, M. G. A. (2019). Fungal-bacterial diversity and microbiome complexity predict ecosystem functioning. Nat. Commun. 10:117. doi: 10.1038/s41467-01912798-y

Wang, X., Pan, Q., Chen, F., Yan, X., and Liao, H. (2011). Effects of co-inoculation with arbuscular mycorrhizal fungi and rhizobia on soybean growth as related to root architecture and availability of $\mathrm{N}$ and P. Mycorrhiza 21, 173-181. doi: 10.1007/s00572-010-0319-1

Ważny, R., Rozpądek, P., Jędrzejczyk, R. J., Śliwa, M., Stojakowska, A., Anielska, T., et al. (2018). Does co-inoculation of Lactuca serriola with endophytic and arbuscular mycorrhizal fungi improve plant growth in a polluted environment? Mycorrhiza 28, 235-246. doi: 10.1007/s00572-018-0819-y

Wei, Z., Gu, Y., Friman, V.-P. P., Kowalchuk, G. A., Xu, Y., Shen, Q., et al. (2019). Initial soil microbiome composition and functioning predetermine future plant health. Sci. Adv. 5:eaaw0759. doi: 10.1126/sciadv.aaw0759

Werner, G. D. A., and Kiers, E. T. (2015). Order of arrival structures arbuscular mycorrhizal colonization of plants. New Phytol. 205, 1515-1524. doi: 10.1111/ nph.13092

Zhu, J., Tremblay, N., and Liang, Y. (2012). Comparing SPAD and atLEAF values for chlorophyll assessment in crop species. Can. J. Soil Sci. 92, 645-648. doi: 10.4141/CJSS2011-100

Conflict of Interest: The authors declare that the research was conducted in the absence of any commercial or financial relationships that could be construed as a potential conflict of interest.

Publisher's Note: All claims expressed in this article are solely those of the authors and do not necessarily represent those of their affiliated organizations, or those of the publisher, the editors and the reviewers. Any product that may be evaluated in this article, or claim that may be made by its manufacturer, is not guaranteed or endorsed by the publisher.

Copyright (c) 2021 Hori, Fujita, Hiruma, Narisawa and Toju. This is an open-access article distributed under the terms of the Creative Commons Attribution License (CC BY). The use, distribution or reproduction in other forums is permitted, provided the original author(s) and the copyright owner(s) are credited and that the original publication in this journal is cited, in accordance with accepted academic practice. No use, distribution or reproduction is permitted which does not comply with these terms. 